Supporting Information for

\title{
Immobilization of "Capping Arene" Cobalt(II) Complexes on Ordered Mesoporous Carbon for Electrocatalytic Water Oxidation
}

\author{
Chang Liu ${ }^{1, \|}$, Ana M. Geer ${ }^{1, \|}$, Christopher Webber ${ }^{1}$, Charles B. Musgrave III $^{2}$, Shunyan $\mathrm{Gu}^{1}$, Grayson \\ Johnson $^{1}$, Diane A. Dickie ${ }^{1}$, Sonia Chabbra ${ }^{3}$, Alexander Schnegg ${ }^{3}$, Hua Zhou ${ }^{4}$, Cheng-Jun Sun ${ }^{4}$, Sooyeon \\ Hwang ${ }^{5}$, William A. Goddard III' ${ }^{2, *}$, Sen Zhang ${ }^{1, *}$, T. Brent Gunnoe ${ }^{1, *}$ \\ 1 Department of Chemistry, University of Virginia, Charlottesville, VA 22904, USA; \\ 2 Materials and Process Simulation Center, Department of Chemistry, California Institute of Technology, Pasadena, \\ CA 91125, USA; \\ 3 EPR Research Group, Max Planck Institute for Chemical Energy Conversion, Stiftstraße 34-36, 45470 Mülheim \\ an der Ruhr, Germany; \\ 4 X-ray Science Division, Advanced Photon Source, Argonne National Laboratory, Lemont, Illinois 60439, USA; \\ 5 Center for Functional Nanomaterials, Brookhaven National Laboratory, Upton, New York 11973, USA
}

॥ These authors contributed equally.

* Corresponding Authors:

Email for W.A.G.: wag@ @altech.edu;ORCID:0000-0003-0097-5716

Email for S.Z.: $\underline{\text { sz3t@ virginia.edu; ORCID: 0000-0002-1716-3741 }}$

Email for T. B. G.: tbg7h@ virginia.edu; ORCID: 0000-0001-5714-3887 


\section{A. Supplementary Methods}

Singe-crystal X-ray diffraction details. A single crystal of (6- $\mathrm{FP}) \mathrm{Co}\left(\mathrm{NO}_{3}\right)_{2}(\mathbf{1})\left(6-\mathrm{FP}=8,8^{\prime}-(1,2-\right.$ phenylene)diquinoline) or (5-FP) $\mathrm{Co}\left(\mathrm{NO}_{3}\right)_{2}$ (2) $(5-\mathrm{FP}=1$,2-bis(N-7-azaindolyl)benzene) was coated with Paratone oil and mounted on a MiTeGen MicroLoop. The X-ray intensity data were measured on a Bruker Kappa APEXII Duo system equipped with a fine-focus sealed tube (Mo K $\alpha$, $\lambda=0.71073 \AA$ ) with a graphite monochromator (for complex 1) and an Incoatec Microfocus $I \mu S$ $(\mathrm{Cu} \mathrm{K} \alpha, \lambda=1.54178 \AA$ ) with a multi-layer mirror monochromator (for complex 2). The frames were integrated with the Bruker SAINT software package ${ }^{1}$ using a narrow-frame algorithm. Data were corrected for absorption effects using the Multi-Scan method (SADABS). ${ }^{1}$ Each structure was solved and refined using the Bruker SHELXTL Software Package ${ }^{2}$ within APEX3 ${ }^{1}$ and OLEX2. ${ }^{3}$ Non-hydrogen atoms were refined anisotropically. Hydrogen atoms were placed in geometrically calculated positions with $U_{i s o}=1.2 U_{\text {equiv }}$ of the parent atom. For complex 2, one solvent site was found to contain dichloromethane disordered over two orientations. The relative occupancy was freely refined, and restraints were used on the anisotropic displacement parameters of the disordered atoms. A second solvent site contained a mixture of $\mathrm{CH}_{2} \mathrm{Cl}_{2}, \mathrm{MeCN}$ and $\mathrm{Et}_{2} \mathrm{O}$ that could not be adequately modeled with or without restraints. Thus, the structure factors were modified using the PLATON SQUEEZE 4 technique, in order to produce a "solvate-free" structure factor set. PLATON reported a total electron density of $140 \mathrm{e}^{-}$and total solvent accessible volume of $368 \AA^{3}$. 


\section{B. Tables S1-5}

Table S1. Crystallographic data for (6-FP)Co( $\left.\mathrm{NO}_{3}\right)_{2}(\mathbf{1})$ and (5-FP)Co( $\left.\mathrm{NO}_{3}\right)_{2}(\mathbf{2})$.

\begin{tabular}{|c|c|c|}
\hline & 1 & 2 \\
\hline CCDC & 2095334 & 2095335 \\
\hline Chemical formula & $\mathrm{C}_{25} \mathrm{H}_{18} \mathrm{Cl}_{2} \mathrm{CoN}_{4} \mathrm{O}_{6}$ & $\mathrm{C}_{81} \mathrm{H}_{57} \mathrm{Cl}_{2} \mathrm{Co}_{4} \mathrm{~N}_{24} \mathrm{O}_{24}$ \\
\hline FW (g/mol) & 600.26 & 2057.12 \\
\hline $\mathbf{T}(\mathbf{K})$ & $100(2)$ & $100(2)$ \\
\hline$\lambda(\AA)$ & 0.71073 & 1.54178 \\
\hline Crystal size (mm) & $0.091 \times 0.105 \times 0.159$ & $0.092 \times 0.151 \times 0.175$ \\
\hline Crystal habit & pink plate & pink plate \\
\hline Crystal system & triclinic & monoclinic \\
\hline Space group & $\mathrm{P}-1$ & $\mathrm{P} 2{ }_{1} / \mathrm{c}$ \\
\hline $\mathbf{a}(\AA)$ & $9.2834(6)$ & $24.9440(8)$ \\
\hline $\mathbf{b}(\AA)$ & $9.3346(6)$ & $10.3178(4)$ \\
\hline c $(\AA)$ & $15.0022(10)$ & $33.2933(10)$ \\
\hline$\alpha\left({ }^{\circ}\right)$ & $91.257(2)$ & 90 \\
\hline$\beta\left(^{\circ}\right)$ & $105.396(2)$ & $93.102(2)$ \\
\hline$\gamma\left({ }^{\circ}\right)$ & $99.442(2)$ & 90 \\
\hline $\mathbf{V}\left(\AA^{3}\right)$ & $1233.47(14)$ & $8556.0(5)$ \\
\hline $\mathbf{Z}$ & 2 & 4 \\
\hline$\rho_{\text {calc }}\left(\mathrm{g} / \mathrm{cm}^{3}\right)$ & 1.616 & 1.597 \\
\hline$\mu\left(\mathbf{m m}^{-1}\right)$ & 0.963 & 7.333 \\
\hline $\mathbf{F ( 0 0 0 )}$ & 610 & 4180 \\
\hline$\theta$ range $\left(^{\circ}\right)$ & 1.41 to 30.53 & 1.77 to 68.48 \\
\hline Index ranges & $\begin{array}{l}-13 \leq \mathrm{h} \leq 13 \\
-13 \leq \mathrm{k} \leq 13 \\
-21 \leq 1 \leq 21\end{array}$ & $\begin{array}{l}-24 \leq \mathrm{h} \leq 30 \\
-12 \leq \mathrm{k} \leq 12 \\
-40 \leq 1 \leq 38\end{array}$ \\
\hline Reflns coll. & 70953 & 62605 \\
\hline Ind. reflns & $7545\left[\mathrm{R}_{\mathrm{int}}=0.0444\right]$ & $15689\left[\mathrm{R}_{\mathrm{int}}=0.1473\right]$ \\
\hline Data / restraints / parameters & $7545 / 0 / 362$ & $15689 / 12 / 1235$ \\
\hline Goodness-of-fit on $F^{2}$ & 1.029 & 1.021 \\
\hline $\mathrm{R}_{\mathbf{1}}[\mathrm{I}>\mathbf{2 \sigma}(\mathrm{I})]$ & 0.0288 & 0.0797 \\
\hline$w_{R_{2}}$ [all data] & 0.0688 & 0.2310 \\
\hline
\end{tabular}

Table S2. Selected bond lengths $(\AA)$ and angles $\left(^{\circ}\right)$ for the four independent molecules in the unit cell of (5-FP) $\mathrm{Co}\left(\mathrm{NO}_{3}\right)_{2}$ (2).

\begin{tabular}{|l|l|l|l|l|}
\hline & $\mathbf{2}$ & $\mathbf{2}$ & $\mathbf{2}$, & $\mathbf{2}$ \\
\hline Co1-N1 & $2.088(6)$ & $2.093(6)$ & $2.056(6)$ & $2.078(6)$ \\
\hline Co1-N4 & $2.124(6)$ & $2.102(5)$ & $2.079(6)$ & $2.081(6)$ \\
\hline Co1-O1 & $2.038(5)$ & $2.038(5)$ & $2.007(5)$ & $2.016(5)$ \\
\hline Co1-O2 & $2.273(5)$ & $2.284(5)$ & $2.282(5)$ & $2.259(5)$ \\
\hline Co1-O4 & $2.072(5)$ & $2.057(5)$ & $2.038(5)$ & $2.016(5)$ \\
\hline Co1-O5 & $2.378(6)$ & $2.381(5)$ & & \\
\hline O1-Co1-N1 & $135.7(2)$ & $168.7(2)$ & $173.4(2)$ & $167.41(19)$ \\
\hline
\end{tabular}


Table S3. DFT free energies at $298 \mathrm{~K}$ for the 6FP structures. Free energies are in Hartree.

\begin{tabular}{|cc|}
\hline Complex & G \\
\hline $6 \mathrm{FP}-\mathrm{Co}(\mathrm{OH})_{2}\left(\mathrm{H}_{2} \mathrm{O}\right)$ & -1406.4775 \\
\hline $6 \mathrm{FP}-\mathrm{Co}(\mathrm{OH})_{3}$ & -1405.8078 \\
\hline $6 \mathrm{FP}-\mathrm{Co}(\mathrm{OH})_{2}(\mathrm{O})$ & -1405.1347 \\
\hline $6 \mathrm{FP}-\mathrm{Co}(\mathrm{OH})_{2}(\mathrm{O})-2 \mathrm{H}_{2} \mathrm{O}-$ path 1 TS & -1557.9686 \\
\hline $6 \mathrm{FP}-\mathrm{Co}(\mathrm{OH})_{2}(\mathrm{O})-2 \mathrm{H}_{2} \mathrm{O}-$ path 2 TS1 & -1557.9565 \\
\hline $6 \mathrm{FP}-\mathrm{Co}(\mathrm{OH})_{2}(\mathrm{O})-2 \mathrm{H}_{2} \mathrm{O}-$ path 2 INT & -1557.9464 \\
\hline $6 \mathrm{FP}-\mathrm{Co}(\mathrm{OH})_{2}(\mathrm{O})-2 \mathrm{H}_{2} \mathrm{O}-$ path 2 TS2 & -1557.9477 \\
\hline $6 \mathrm{FP}-\mathrm{Co}(\mathrm{OH})_{2}(\mathrm{O})-2 \mathrm{H}_{2} \mathrm{O}-$ path 3 TS & -1557.9452 \\
\hline $6 \mathrm{FP}-\mathrm{Co}(\mathrm{OH})_{2}(\mathrm{O})-2 \mathrm{H}_{2} \mathrm{O}-$ path $4 \mathrm{TS}$ & -1557.4770 \\
\hline
\end{tabular}

Table S4. DFT free energies at $298 \mathrm{~K}$ for the 5FP structures. Free energies are in Hartree.

\begin{tabular}{|cc|}
\hline Complex & G \\
\hline $5 \mathrm{FP}-\mathrm{Co}(\mathrm{OH})_{2}\left(\mathrm{H}_{2} \mathrm{O}\right)$ & -1362.3857 \\
\hline $5 \mathrm{FP}-\mathrm{Co}(\mathrm{OH})_{3}$ & -1361.7189 \\
\hline $5 \mathrm{FP}-\mathrm{Co}(\mathrm{OH})_{2}(\mathrm{O})$ & -1361.0427 \\
\hline $5 \mathrm{FP}-\mathrm{Co}(\mathrm{OH})_{2}(\mathrm{O})-2 \mathrm{H}_{2} \mathrm{O}-$ path 1 TS & -1513.8372 \\
\hline $5 \mathrm{FP}-\mathrm{Co}(\mathrm{OH})_{2}(\mathrm{O})-2 \mathrm{H}_{2} \mathrm{O}-$ path 2 TS1 & -1513.8674 \\
\hline $5 \mathrm{FP}-\mathrm{Co}(\mathrm{OH})_{2}(\mathrm{O})-2 \mathrm{H}_{2} \mathrm{O}-$ path 2 INT & -1513.8574 \\
\hline $5 \mathrm{FP}-\mathrm{Co}(\mathrm{OH})_{2}(\mathrm{O})-2 \mathrm{H}_{2} \mathrm{O}-$ path 2 TS2 & -1513.8521 \\
\hline $5 \mathrm{FP}-\mathrm{Co}(\mathrm{OH})_{2}(\mathrm{O})-2 \mathrm{H}_{2} \mathrm{O}-$ path 3 TS & -1513.8480 \\
\hline $5 \mathrm{FP}-\mathrm{Co}(\mathrm{OH})_{2}(\mathrm{O})-2 \mathrm{H}_{2} \mathrm{O}-$ path 4 TS & -1513.4100 \\
\hline
\end{tabular}

Table S5. DFT-optimized coordinates

\section{FP-Co $(\mathrm{OH})_{2}\left(\mathrm{H}_{2} \mathrm{O}\right)$}

Co1 3.1115772534

$\mathrm{O} 2 \quad 4.8275097127$

O3 $\quad 2.4068208366$

O50 $\quad 3.9449863098$

H7 $\quad 3.2825604481$

H8 $\quad 4.6483566869$
7.3248436409

7.3295647376

6.5679492926

4.8661353034

5.0671881040

5.4922390140
13.5341438728

12.7225328580

11.9362653528

13.3611593788

12.6633785694

13.0812695379 


\begin{tabular}{|c|c|c|c|}
\hline N9 & 1.3642883210 & 7.0233104197 & 14.4578890985 \\
\hline N10 & 3.8737647381 & 7.7983831014 & 15.3135563524 \\
\hline C11 & 1.2430588903 & 5.8149186207 & 14.9896869204 \\
\hline $\mathrm{C} 12$ & 0.1159714860 & 5.4070558215 & 15.7263150572 \\
\hline $\mathrm{C} 13$ & -0.9171740565 & 6.2911256419 & 15.9017813000 \\
\hline $\mathrm{C} 14$ & -0.8383002082 & 7.5822052315 & 15.3286743246 \\
\hline C15 & 0.3398041179 & 7.9257034239 & 14.5955216686 \\
\hline C16 & -1.8910102248 & 8.5236584006 & 15.4419873764 \\
\hline $\mathrm{C} 17$ & -1.7892573894 & 9.7486805404 & 14.8345768876 \\
\hline $\mathrm{C} 18$ & -0.6307361483 & 10.0802613902 & 14.0998461535 \\
\hline C19 & 0.4313162713 & 9.2074661536 & 13.9759320527 \\
\hline $\mathrm{C} 20$ & 1.6228228543 & 9.6076885110 & 13.1741381505 \\
\hline $\mathrm{C} 21$ & 1.5259557221 & 9.6573351612 & 11.7777461486 \\
\hline $\mathrm{C} 22$ & 2.6038119808 & 10.0609377119 & 11.0033418787 \\
\hline $\mathrm{C} 23$ & 3.7951195244 & 10.4404504477 & 11.6147773836 \\
\hline $\mathrm{C} 24$ & 3.9004251810 & 10.4149550589 & 12.9980991096 \\
\hline $\mathrm{C} 25$ & 2.8276759836 & 9.9923016904 & 13.7936555877 \\
\hline $\mathrm{C} 26$ & 2.9753473948 & 10.0386353455 & 15.2799378248 \\
\hline $\mathrm{C} 27$ & 2.6095860580 & 11.1676685905 & 15.9831273652 \\
\hline $\mathrm{C} 28$ & 2.8298678369 & 11.2795680052 & 17.3732870045 \\
\hline $\mathrm{C} 29$ & 3.4487172421 & 10.2646711087 & 18.0588723460 \\
\hline C30 & 3.8422141061 & 9.0838348720 & 17.3822348421 \\
\hline C31 & 3.5704324485 & 8.9553155069 & 15.9868431845 \\
\hline C32 & 4.5056256294 & 8.0147861868 & 18.0313953509 \\
\hline C33 & 4.8568877680 & 6.9002562811 & 17.3133989253 \\
\hline C34 & 4.5059967743 & 6.8245613445 & 15.9514175838 \\
\hline H35 & 2.0769001932 & 5.1427695215 & 14.8144766256 \\
\hline H36 & 0.0856835849 & 4.4053619162 & 16.1351732829 \\
\hline H37 & -1.8030276746 & 6.0143010000 & 16.4631923460 \\
\hline H38 & -2.7769253445 & 8.2522635907 & 16.0054810345 \\
\hline H39 & -2.5961311626 & 10.4679203739 & 14.9121056706 \\
\hline $\mathrm{H} 40$ & -0.5624139289 & 11.0492628920 & 13.6191426095 \\
\hline $\mathrm{H} 41$ & 0.6046434961 & 9.3388952420 & 11.3058073913 \\
\hline $\mathrm{H} 42$ & 2.5227231033 & 10.0544273193 & 9.9234487768 \\
\hline $\mathrm{H} 43$ & 4.6470879368 & 10.7355952629 & 11.0148868015 \\
\hline $\mathrm{H} 44$ & 4.8268577596 & 10.7030941201 & 13.4808397581 \\
\hline $\mathrm{H} 45$ & 2.1570855124 & 11.9923532944 & 15.4450879097 \\
\hline H46 & 2.5248958079 & 12.1819498986 & 17.8901311716 \\
\hline H47 & 3.6482445291 & 10.3509611192 & 19.1213509097 \\
\hline $\mathrm{H} 48$ & 4.7302126698 & 8.0940447693 & 19.0896199172 \\
\hline H49 & 5.3737844099 & 6.0671323591 & 17.7720638200 \\
\hline $\mathrm{H} 50$ & 4.7046798552 & 5.9359432261 & 15.3641883895 \\
\hline H51 & 1.4452808529 & 6.5974748945 & 11.9191286723 \\
\hline H52 & 5.4300561391 & 7.9270217477 & 13.1752343269 \\
\hline \multicolumn{4}{|c|}{$6 \mathrm{FP}-\mathrm{Co}(\mathrm{OH})_{3}$} \\
\hline Co1 & 3.0330509689 & 7.2493260946 & 13.4590437880 \\
\hline $\mathrm{O} 2$ & 4.4737788209 & 7.5416408955 & 12.3481187515 \\
\hline $\mathrm{O} 3$ & 1.9276041823 & 6.8742245593 & 12.0267871982 \\
\hline O50 & 3.5112453289 & 5.4779143638 & 13.6777185176 \\
\hline H7 & 4.1184885936 & 5.3587037288 & 12.9352050668 \\
\hline N9 & 1.3864790753 & 7.0380374563 & 14.6280254958 \\
\hline N10 & 4.0285121917 & 7.7704261967 & 15.2284118434 \\
\hline $\mathrm{C} 11$ & 1.2655647204 & 5.9080214946 & 15.2995037931 \\
\hline $\mathrm{C} 12$ & 0.1182226531 & 5.5780859069 & 16.0464517310 \\
\hline $\mathrm{C} 13$ & -0.9353328675 & 6.4532079205 & 16.0633322649 \\
\hline
\end{tabular}




\begin{tabular}{|c|c|c|c|}
\hline $\mathrm{C} 14$ & -0.8487414682 & 7.6665195417 & 15.3405960884 \\
\hline $\mathrm{C} 15$ & 0.3582366921 & 7.9421358323 & 14.6275787741 \\
\hline $\mathrm{C} 16$ & -1.9196219778 & 8.5924621473 & 15.2935153922 \\
\hline $\mathrm{C} 17$ & -1.8027901652 & 9.7429955346 & 14.5573542936 \\
\hline $\mathrm{C} 18$ & -0.6056440792 & 10.0175929258 & 13.8630175469 \\
\hline C19 & 0.4743940568 & 9.1576052968 & 13.8934969860 \\
\hline $\mathrm{C} 20$ & 1.7134537141 & 9.5463710900 & 13.1603797218 \\
\hline $\mathrm{C} 21$ & 1.6827289249 & 9.6374564345 & 11.7580618177 \\
\hline $\mathrm{C} 22$ & 2.7664114670 & 10.1302007007 & 11.0554461627 \\
\hline $\mathrm{C} 23$ & 3.9034672833 & 10.5635557583 & 11.7374135685 \\
\hline $\mathrm{C} 24$ & 3.9506917870 & 10.4875238269 & 13.1182617617 \\
\hline $\mathrm{C} 25$ & 2.8694885493 & 9.9667232050 & 13.8440955589 \\
\hline $\mathrm{C} 26$ & 2.9815698979 & 9.9426089350 & 15.3304534301 \\
\hline $\mathrm{C} 27$ & 2.5289751953 & 10.9950089649 & 16.0977850166 \\
\hline $\mathrm{C} 28$ & 2.7651487995 & 11.0500166108 & 17.4885638765 \\
\hline $\mathrm{C} 29$ & 3.4941716455 & 10.0632891629 & 18.1040158916 \\
\hline C30 & 3.9740453943 & 8.9596453767 & 17.3568447453 \\
\hline C31 & 3.6729319030 & 8.8739539503 & 15.9646279455 \\
\hline C32 & 4.7558790258 & 7.9277976499 & 17.9300407573 \\
\hline C33 & 5.1763301009 & 6.8857157073 & 17.1460483493 \\
\hline C34 & 4.7611722082 & 6.8304712146 & 15.7993111671 \\
\hline H35 & 2.1031462606 & 5.2276785641 & 15.2037425069 \\
\hline H36 & 0.0843683105 & 4.6347351861 & 16.5763374821 \\
\hline H37 & -1.8431906060 & 6.2290179786 & 16.6133462014 \\
\hline H38 & -2.8296454793 & 8.3684303799 & 15.8392825143 \\
\hline H39 & -2.6226142779 & 10.4501357080 & 14.5087233493 \\
\hline $\mathrm{H} 40$ & -0.5181635317 & 10.9365040032 & 13.2947453138 \\
\hline $\mathrm{H} 41$ & 0.8150498073 & 9.2585100930 & 11.2372228082 \\
\hline $\mathrm{H} 42$ & 2.7391989181 & 10.1524240846 & 9.9730028447 \\
\hline $\mathrm{H} 43$ & 4.7584301977 & 10.9386464134 & 11.1876292831 \\
\hline $\mathrm{H} 44$ & 4.8352678551 & 10.8075458852 & 13.6564296061 \\
\hline H45 & 1.9998138208 & 11.8057360077 & 15.6110513007 \\
\hline $\mathrm{H} 46$ & 2.3913900158 & 11.8913149372 & 18.0602296256 \\
\hline $\mathrm{H} 47$ & 3.7160570373 & 10.1140400572 & 19.1643565612 \\
\hline $\mathrm{H} 48$ & 5.0116090822 & 7.9779813146 & 18.9831657932 \\
\hline H49 & 5.7838753870 & 6.0830598730 & 17.5443478173 \\
\hline H50 & 4.9501534431 & 5.9685967082 & 15.1700011396 \\
\hline H51 & 2.0864926051 & 5.9491536059 & 11.8118374545 \\
\hline H52 & 5.2215043339 & 7.7919086015 & 12.9035348703 \\
\hline \multicolumn{4}{|c|}{$6 \mathrm{FP}-\mathrm{Co}(\mathrm{OH})_{2}(\mathrm{O})$} \\
\hline Co1 & 3.2092640750 & 7.2027212715 & 13.4913678600 \\
\hline $\mathrm{O} 2$ & 4.7701129372 & 7.4497288634 & 12.5521607935 \\
\hline $\mathrm{O} 3$ & 2.2463460316 & 7.0197915601 & 11.9785054949 \\
\hline O50 & 3.5951957416 & 5.5510516124 & 13.8170387183 \\
\hline N9 & 1.3467149419 & 7.1259641927 & 14.5931194798 \\
\hline N10 & 4.0989197611 & 7.7560924262 & 15.2687277295 \\
\hline $\mathrm{C} 11$ & 1.2356753779 & 5.9958642534 & 15.2705469016 \\
\hline $\mathrm{C} 12$ & 0.0954444482 & 5.6277719366 & 16.0088904772 \\
\hline $\mathrm{C} 13$ & -0.9826287169 & 6.4683230762 & 16.0146382736 \\
\hline $\mathrm{C} 14$ & -0.9203750996 & 7.6838998500 & 15.2944076398 \\
\hline $\mathrm{C} 15$ & 0.2867082010 & 8.0051519898 & 14.5916160345 \\
\hline $\mathrm{C} 16$ & -2.0288873451 & 8.5650481778 & 15.2560898561 \\
\hline $\mathrm{C} 17$ & -1.9611012254 & 9.7315396440 & 14.5409685237 \\
\hline $\mathrm{C} 18$ & -0.7723225900 & 10.0621566012 & 13.8624061094 \\
\hline C19 & 0.3438804134 & 9.2468980957 & 13.8829396486 \\
\hline
\end{tabular}




$\begin{array}{lccc}\text { C20 } & 1.5558660237 & 9.6823337336 & 13.1542433960 \\ \text { C21 } & 1.4870562312 & 9.9056495401 & 11.7781597544 \\ \text { C22 } & 2.6226151388 & 10.2490661418 & 11.0565670832 \\ \text { C23 } & 3.8517469854 & 10.3589758361 & 11.7001510792 \\ \text { C24 } & 3.9300517342 & 10.1641394234 & 13.0712036001 \\ \text { C25 } & 2.7867813678 & 9.8409419829 & 13.8176178141 \\ \text { C26 } & 2.8939662894 & 9.8361507046 & 15.3063444678 \\ \text { C27 } & 2.3442268908 & 10.8577843193 & 16.0533539687 \\ \text { C28 } & 2.5730362158 & 10.9643175546 & 17.4424229770 \\ \text { C29 } & 3.3927361784 & 10.0668669361 & 18.0812061174 \\ \text { C30 } & 3.9678063761 & 8.9920405021 & 17.3596662426 \\ \text { C31 } & 3.6660682169 & 8.8463402697 & 15.9731196102 \\ \text { C32 } & 4.8388903993 & 8.0433319017 & 17.9473689736 \\ \text { C33 } & 5.3439851646 & 7.0230004258 & 17.1828106183 \\ \text { C34 } & 4.9227929833 & 6.9001301969 & 15.8429984734 \\ \text { H35 } & 2.0918218490 & 5.3360984435 & 15.1838396286 \\ \text { H36 } & 0.0900651949 & 4.6834426215 & 16.5382628024 \\ \text { H37 } & -1.8905235032 & 6.2195224068 & 16.5540785306 \\ \text { H38 } & -2.9286737377 & 8.2926130177 & 15.7968505332 \\ \text { H39 } & -2.8082406393 & 10.4062257706 & 14.5018943540 \\ \text { H40 } & -0.7139065119 & 10.9964435042 & 13.3158902466 \\ \text { H41 } & 0.5458184228 & 9.7427636229 & 11.2688525668 \\ \text { H42 } & 2.5553429940 & 10.3916823675 & 9.9841735677 \\ \text { H43 } & 4.7507612057 & 10.5761792407 & 11.1372836887 \\ \text { H44 } & 4.8827474810 & 10.2418636412 & 13.5783644577 \\ \text { H45 } & 1.7486326898 & 11.6098383804 & 15.5497640567 \\ \text { H46 } & 2.1242793834 & 11.7821738325 & 17.9938947134 \\ \text { H47 } & 3.6120550640 & 10.1667657123 & 19.1385790979 \\ \text { H48 } & 5.0982827893 & 8.1388864164 & 18.9964547152 \\ \text { H49 } & 6.0249507845 & 6.2882466802 & 17.5928974659 \\ \text { H50 } & 5.2234636218 & 6.0626721455 & 15.2249708878 \\ \text { H51 } & 2.9144650746 & 7.0014788023 & 11.2812710710 \\ \text { H52 } & 5.1253649483 & 6.5567631709 & 12.4605003409\end{array}$

$\begin{array}{lccc}\mathbf{6 F P}-\mathbf{C o}(\mathbf{O H})_{\mathbf{2}}(\mathbf{O})-\mathbf{2}\left(\mathbf{H}_{\mathbf{2}} \mathbf{O}\right) & - \text { Path } \mathbf{1} \text { TS } & \\ \text { Co1 } & 3.1876323829 & 7.2801207603 & 13.5520564332 \\ \text { O2 } & 4.7740905684 & 7.6725130636 & 12.7031225919 \\ \text { O3 } & 2.5988710482 & 6.2301234307 & 12.0129252944 \\ \text { O50 } & 3.3902823911 & 5.3492456767 & 13.3635440200 \\ \text { N9 } & 1.3865184691 & 7.0401860682 & 14.5390585785 \\ \text { N10 } & 3.9850760374 & 7.8208906009 & 15.2782751030 \\ \text { C11 } & 1.2859597978 & 5.9054012011 & 15.2164107859 \\ \text { C12 } & 0.1584788389 & 5.5509755158 & 15.9808048862 \\ \text { C13 } & -0.9123928401 & 6.4036854765 & 16.0165035023 \\ \text { C14 } & -0.8583273871 & 7.6200597347 & 15.2966722655 \\ \text { C15 } & 0.3359264497 & 7.9266781781 & 14.5708010377 \\ \text { C16 } & -1.9508850299 & 8.5211016756 & 15.2692673023 \\ \text { C17 } & -1.8703182168 & 9.6815935152 & 14.5439285358 \\ \text { C18 } & -0.6857916665 & 9.9930052033 & 13.8449286196 \\ \text { C19 } & 0.4142920486 & 9.1578368411 & 13.8567044430 \\ \text { C20 } & 1.6466505369 & 9.5780110931 & 13.1375972736 \\ \text { C21 } & 1.6315274136 & 9.6829379190 & 11.7407996941 \\ \text { C22 } & 2.7426551171 & 10.1495331947 & 11.0524631168 \\ \text { C23 } & 3.8830462422 & 10.5310286415 & 11.7530166327 \\ \text { C24 } & 3.9055257754 & 10.4492470305 & 13.1370123766 \\ \text { C25 } & 2.7980919620 & 9.9729546809 & 13.8473673391\end{array}$




\begin{tabular}{|c|c|c|c|}
\hline $\mathrm{C} 26$ & 2.8759312021 & 9.9553408085 & 15.3409394755 \\
\hline $\mathrm{C} 27$ & 2.3842933904 & 10.9967994200 & 16.0984715607 \\
\hline $\mathrm{C} 28$ & 2.5987454820 & 11.0606730378 & 17.4941423855 \\
\hline C29 & 3.3498941102 & 10.1018015700 & 18.1272379297 \\
\hline C30 & 3.8756005796 & 9.0099625310 & 17.3926123921 \\
\hline C31 & 3.5855419500 & 8.9110271564 & 16.0005177176 \\
\hline C32 & 4.6940345687 & 8.0065672540 & 17.9668058371 \\
\hline C33 & 5.1705356622 & 6.9858636513 & 17.1825524728 \\
\hline C34 & 4.7714540979 & 6.9168883275 & 15.8320928823 \\
\hline H35 & 2.1301626949 & 5.2365806679 & 15.0986482465 \\
\hline H36 & 0.1552073603 & 4.6076287660 & 16.5115962545 \\
\hline H37 & -1.8067090635 & 6.1613557383 & 16.5805512834 \\
\hline $\mathrm{H} 38$ & -2.8481386763 & 8.2708276579 & 15.8244142924 \\
\hline H39 & -2.7067802271 & 10.3697684891 & 14.5125959690 \\
\hline $\mathrm{H} 40$ & -0.6199018998 & 10.9237567576 & 13.2935277905 \\
\hline $\mathrm{H} 41$ & 0.7467196797 & 9.3651891977 & 11.2025048207 \\
\hline $\mathrm{H} 42$ & 2.7261535612 & 10.1941319184 & 9.9700636041 \\
\hline $\mathrm{H} 43$ & 4.7647494306 & 10.8664651408 & 11.2215812760 \\
\hline H44 & 4.8018447730 & 10.7152216697 & 13.6827194715 \\
\hline $\mathrm{H} 45$ & 1.8460720416 & 11.7961933045 & 15.6029037721 \\
\hline $\mathrm{H} 46$ & 2.1929314016 & 11.8937415621 & 18.0559670290 \\
\hline $\mathrm{H} 47$ & 3.5568569786 & 10.1685680438 & 19.1896165563 \\
\hline $\mathrm{H} 48$ & 4.9422857938 & 8.0640319515 & 19.0211087421 \\
\hline $\mathrm{H} 49$ & 5.8179135640 & 6.2169983028 & 17.5840877154 \\
\hline $\mathrm{H} 50$ & 5.0583013274 & 6.0973531296 & 15.1819625329 \\
\hline H51 & 1.7341767191 & 5.8312688454 & 12.1693133066 \\
\hline H52 & 4.9018011023 & 6.9626652712 & 12.0639995859 \\
\hline H54 & 6.8450612952 & 5.6745076910 & 13.1925110632 \\
\hline O52 & 6.9257152023 & 6.3658513828 & 13.8641480941 \\
\hline H55 & 6.2789993630 & 7.0207538522 & 13.5244257784 \\
\hline H53 & 5.1627899347 & 4.7576388736 & 10.9407531255 \\
\hline $\mathrm{O} 53$ & 5.5258036463 & 4.8240951072 & 11.8275907566 \\
\hline H56 & 4.7332663637 & 4.9315388335 & 12.4087328885 \\
\hline \multicolumn{4}{|c|}{$6 \mathrm{FP}-\mathrm{Co}(\mathrm{OH})_{2}(\mathrm{O})-2\left(\mathrm{H}_{2} \mathrm{O}\right)$ - Path 2 TS1 } \\
\hline Co1 & 3.1098217146 & 7.2284835177 & 13.3519874020 \\
\hline $\mathrm{O} 2$ & 4.6006804719 & 7.6093026032 & 12.3845573968 \\
\hline $\mathrm{O} 3$ & 2.0498247953 & 6.8925736082 & 11.8320908322 \\
\hline $\mathrm{O} 50$ & 3.5119670282 & 5.5202874110 & 13.5624689894 \\
\hline N9 & 1.3709223873 & 7.0473848269 & 14.4989835199 \\
\hline N10 & 4.0978156330 & 7.6636204910 & 15.0525827863 \\
\hline $\mathrm{C} 11$ & 1.2561968121 & 5.9165915516 & 15.1717300996 \\
\hline $\mathrm{C} 12$ & 0.1000967352 & 5.5481339700 & 15.8828526308 \\
\hline C13 & -0.9881996659 & 6.3748399970 & 15.8453448579 \\
\hline $\mathrm{C} 14$ & -0.9197644548 & 7.5860631199 & 15.1170288345 \\
\hline C15 & 0.3068834918 & 7.9146397600 & 14.4608968769 \\
\hline C16 & -2.0305773712 & 8.4582924345 & 15.0163373893 \\
\hline C17 & -1.9366205743 & 9.6155735286 & 14.2870432599 \\
\hline $\mathrm{C} 18$ & -0.7202980453 & 9.9518661263 & 13.6601512488 \\
\hline C19 & 0.3986313218 & 9.1441929759 & 13.7449020913 \\
\hline $\mathrm{C} 20$ & 1.6555975403 & 9.5984027513 & 13.0972666190 \\
\hline $\mathrm{C} 21$ & 1.6677939042 & 9.8501694392 & 11.7171463314 \\
\hline $\mathrm{C} 22$ & 2.7986827113 & 10.3517888922 & 11.0960188511 \\
\hline $\mathrm{C} 23$ & 3.9459849157 & 10.6176569456 & 11.8426687262 \\
\hline $\mathrm{C} 24$ & 3.9498229714 & 10.3805116149 & 13.2053288779 \\
\hline $\mathrm{C} 25$ & 2.8140583126 & 9.8672176863 & 13.8508482403 \\
\hline
\end{tabular}




$\begin{array}{lccc}\text { C26 } & 2.8831622086 & 9.7169446051 & 15.3351171348 \\ \text { C27 } & 2.3374537364 & 10.6473455984 & 16.1930158040 \\ \text { C28 } & 2.5865404793 & 10.5980933947 & 17.5833505619 \\ \text { C29 } & 3.4309563274 & 9.6492946813 & 18.1063189834 \\ \text { C30 } & 4.0085317546 & 8.6680181518 & 17.2628712842 \\ \text { C31 } & 3.6699313914 & 8.6670806799 & 15.8803878715 \\ \text { C32 } & 4.9256261080 & 7.6872293932 & 17.7143946546 \\ \text { C33 } & 5.4429009832 & 6.7805412967 & 16.8244126658 \\ \text { C34 } & 4.9765805016 & 6.7831668860 & 15.4931625108 \\ \text { H35 } & 2.1096163192 & 5.2551445398 & 15.0952447082 \\ \text { H36 } & 0.0846330221 & 4.6045001092 & 16.4121827940 \\ \text { H37 } & -1.9108838854 & 6.1148948884 & 16.3531603872 \\ \text { H38 } & -2.9529824886 & 8.1884578292 & 15.5186302303 \\ \text { H39 } & -2.7865708256 & 10.2818873210 & 14.1990623173 \\ \text { H40 } & -0.6441935884 & 10.8820139372 & 13.1089545739 \\ \text { H41 } & 0.7897256483 & 9.5996581345 & 11.1367139707 \\ \text { H42 } & 2.7975955960 & 10.5106154088 & 10.0242452750 \\ \text { H43 } & 4.8417386352 & 10.9846514678 & 11.3573173857 \\ \text { H44 } & 4.8437673676 & 10.5617034647 & 13.7885653076 \\ \text { H45 } & 1.7350412361 & 11.4496030753 & 15.7835798104 \\ \text { H46 } & 2.1388870361 & 11.3438320139 & 18.2296459796 \\ \text { H47 } & 3.6718884826 & 9.6411060187 & 19.1635356972 \\ \text { H48 } & 5.2162672774 & 7.6731003677 & 18.7593974232 \\ \text { H49 } & 6.1654366536 & 6.0344942717 & 17.1286935525 \\ \text { H50 } & 5.2773921307 & 6.0330095324 & 14.7713057314 \\ \text { H51 } & 2.5466787349 & 6.1918510075 & 11.3322656371 \\ \text { H52 } & 4.6840117058 & 6.9465616375 & 11.6886765913 \\ \text { O49 } & 2.7618191087 & 4.4134006458 & 11.2273849837 \\ \text { H53 } & 3.3856577405 & 4.1940043945 & 11.9298138723 \\ \text { H54 } & 1.6488998104 & 4.4769476682 & 11.8147285368 \\ \text { O52 } & 0.6083689679 & 4.9428984266 & 12.3511849936 \\ \text { H55 } & -0.1080991023 & 4.8502758227 & 11.7147247563 \\ \text { H56 } & 1.1643073219 & 6.0565316096 & 12.1553439615\end{array}$

$\begin{array}{lccc}\mathbf{6 F P}-\mathbf{C o}(\mathbf{O H})_{\mathbf{2}}(\mathbf{O}) \mathbf{- 2}\left(\mathbf{H}_{\mathbf{2}} \mathbf{O}\right) & - \text { Path } 2 \text { Intermediate } & \\ \text { Co1 } & 3.1945292806 & 7.3649604179 & 13.4029250874 \\ \text { O2 } & 4.6730906579 & 7.8422796105 & 12.3809947292 \\ \text { O3 } & 2.2962775548 & 7.0698342842 & 11.7054832173 \\ \text { O50 } & 3.8193537953 & 5.6792523456 & 13.3993462580 \\ \text { N9 } & 1.4731383095 & 7.0333389172 & 14.4345074571 \\ \text { N10 } & 4.0460022339 & 7.7147211851 & 15.1708269181 \\ \text { C11 } & 1.4170777560 & 5.8725366613 & 15.0703711642 \\ \text { C12 } & 0.2845366259 & 5.4500766016 & 15.7947790299 \\ \text { C13 } & -0.8164299715 & 6.2577365250 & 15.8437745190 \\ \text { C14 } & -0.7954045642 & 7.5074478923 & 15.1778224686 \\ \text { C15 } & 0.3930701148 & 7.8862000196 & 14.4809335013 \\ \text { C16 } & -1.9187980223 & 8.3687128263 & 15.1794450345 \\ \text { C17 } & -1.8757341796 & 9.5666136458 & 14.5132283690 \\ \text { C18 } & -0.6978052342 & 9.9502223497 & 13.8435949215 \\ \text { C19 } & 0.4308105722 & 9.1514219747 & 13.8259128735 \\ \text { C20 } & 1.6420220291 & 9.6510964875 & 13.1252132450 \\ \text { C21 } & 1.5693278113 & 9.9356819447 & 11.7528454384 \\ \text { C22 } & 2.6528473119 & 10.4753938193 & 11.0801697338 \\ \text { C23 } & 3.8401624969 & 10.7382949020 & 11.7640049838 \\ \text { C24 } & 3.9249502597 & 10.4754478370 & 13.1192672506 \\ \text { C25 } & 2.8329486060 & 9.9383014095 & 13.8208351439\end{array}$




\begin{tabular}{|c|c|c|c|}
\hline $\mathrm{C} 26$ & 2.9438158841 & 9.8544099851 & 15.3087105979 \\
\hline $\mathrm{C} 27$ & 2.4528866451 & 10.8718257008 & 16.1006425048 \\
\hline $\mathrm{C} 28$ & 2.6834667436 & 10.9016500906 & 17.4926055944 \\
\hline $\mathrm{C} 29$ & 3.4424714030 & 9.9244091183 & 18.0874896903 \\
\hline C30 & 3.9557799539 & 8.8524255927 & 17.3175464960 \\
\hline C31 & 3.6632836202 & 8.7953121581 & 15.9231506010 \\
\hline C32 & 4.7510775087 & 7.8191563979 & 17.8702717159 \\
\hline C33 & 5.1880255851 & 6.7992100553 & 17.0667041574 \\
\hline C34 & 4.7838065731 & 6.7646300580 & 15.7162366674 \\
\hline H35 & 2.2638731129 & 5.2036480146 & 14.9269103709 \\
\hline H36 & 0.3093951892 & 4.4781899473 & 16.2699745756 \\
\hline H37 & -1.7143569809 & 5.9581797764 & 16.3742602341 \\
\hline H38 & -2.8117403802 & 8.0579393921 & 15.7107840973 \\
\hline H39 & -2.7359829480 & 10.2254919692 & 14.5063006352 \\
\hline $\mathrm{H} 40$ & -0.6590970290 & 10.9086615258 & 13.3387311035 \\
\hline $\mathrm{H} 41$ & 0.6598033569 & 9.6890209841 & 11.2202810593 \\
\hline $\mathrm{H} 42$ & 2.5830577273 & 10.6706013385 & 10.0162138192 \\
\hline $\mathrm{H} 43$ & 4.7012361029 & 11.1292741001 & 11.2364181742 \\
\hline H44 & 4.8450742008 & 10.6648926972 & 13.6563206266 \\
\hline H45 & 1.8994955901 & 11.6768294640 & 15.6315966692 \\
\hline $\mathrm{H} 46$ & 2.2813162675 & 11.7169505981 & 18.0823205381 \\
\hline $\mathrm{H} 47$ & 3.6585264930 & 9.9565479320 & 19.1497220773 \\
\hline $\mathrm{H} 48$ & 4.9991670532 & 7.8482189348 & 18.9260491944 \\
\hline H49 & 5.7998790155 & 5.9936971608 & 17.4519231441 \\
\hline H50 & 4.9773918471 & 5.9261699872 & 15.0537730380 \\
\hline H51 & 3.1121793029 & 7.0839902477 & 11.1779307304 \\
\hline H52 & 5.2362049096 & 7.0589382233 & 12.3870848667 \\
\hline O49 & 2.6947641502 & 3.7687286382 & 13.7156911460 \\
\hline H53 & 3.5826334515 & 3.6606018821 & 13.3588898307 \\
\hline H54 & 1.8055083771 & 4.3336550555 & 12.5786474617 \\
\hline O52 & 1.2402104444 & 4.7581525432 & 11.8855816934 \\
\hline H55 & 1.2532690585 & 4.1536471027 & 11.1270946110 \\
\hline H56 & 1.9123750910 & 6.1216045526 & 11.7000454572 \\
\hline \multicolumn{4}{|c|}{$6 \mathrm{FP}-\mathrm{Co}(\mathrm{OH})_{2}(\mathrm{O})-2\left(\mathrm{H}_{2} \mathrm{O}\right)-$ Path 2 TS2 } \\
\hline Col & 3.2013564903 & 7.3427470671 & 13.4235747362 \\
\hline $\mathrm{O} 2$ & 4.7020890078 & 7.7301372969 & 12.3783445884 \\
\hline $\mathrm{O} 3$ & 2.2983374403 & 7.0884064927 & 11.7069756161 \\
\hline O50 & 3.7795944615 & 5.5964928581 & 13.4820917324 \\
\hline N9 & 1.4548571609 & 7.0689475258 & 14.4545104065 \\
\hline N10 & 4.0712936069 & 7.7147471001 & 15.1767263523 \\
\hline $\mathrm{C} 11$ & 1.3900146001 & 5.9031055636 & 15.0823752538 \\
\hline $\mathrm{C} 12$ & 0.2528217521 & 5.4720289766 & 15.7914698827 \\
\hline $\mathrm{C} 13$ & -0.8512641341 & 6.2773688083 & 15.8300008392 \\
\hline $\mathrm{C} 14$ & -0.8255192420 & 7.5295150122 & 15.1706734170 \\
\hline $\mathrm{C} 15$ & 0.3707442022 & 7.9163099183 & 14.4891690905 \\
\hline $\mathrm{C} 16$ & -1.9537636092 & 8.3853579971 & 15.1649598167 \\
\hline $\mathrm{C} 17$ & -1.9092370823 & 9.5856970860 & 14.5042295713 \\
\hline $\mathrm{C} 18$ & -0.7266170403 & 9.9767935090 & 13.8466163144 \\
\hline C19 & 0.4072743686 & 9.1856840691 & 13.8357261277 \\
\hline $\mathrm{C} 20$ & 1.6169012541 & 9.6867688972 & 13.1356083659 \\
\hline $\mathrm{C} 21$ & 1.5434258058 & 9.9760769992 & 11.7670364899 \\
\hline $\mathrm{C} 22$ & 2.6448723228 & 10.4685327796 & 11.0831899098 \\
\hline $\mathrm{C} 23$ & 3.8491843902 & 10.6710840727 & 11.7539338360 \\
\hline $\mathrm{C} 24$ & 3.9332714643 & 10.4064062629 & 13.1109482183 \\
\hline $\mathrm{C} 25$ & 2.8241142156 & 9.9240570178 & 13.8220623057 \\
\hline
\end{tabular}

S10 


\begin{tabular}{|c|c|c|c|}
\hline $\mathrm{C} 26$ & 2.9374260362 & 9.8394602696 & 15.3094683894 \\
\hline $\mathrm{C} 27$ & 2.4248201189 & 10.8457060755 & 16.1022386468 \\
\hline $\mathrm{C} 28$ & 2.6591827123 & 10.8836487186 & 17.4934176035 \\
\hline $\mathrm{C} 29$ & 3.4454602638 & 9.9275298380 & 18.0871209586 \\
\hline C30 & 3.9792281835 & 8.8651599415 & 17.3176267677 \\
\hline C31 & 3.6785758207 & 8.7946212892 & 15.9253124497 \\
\hline C32 & 4.8022387679 & 7.8531965041 & 17.8693168185 \\
\hline C33 & 5.2546488832 & 6.8384176098 & 17.0675255144 \\
\hline C34 & 4.8362007155 & 6.7854722929 & 15.7217734434 \\
\hline H35 & 2.2555542411 & 5.2595349490 & 14.9690957843 \\
\hline H36 & 0.2734968155 & 4.5001824520 & 16.2669019722 \\
\hline H37 & -1.7543852056 & 5.9728536112 & 16.3485552036 \\
\hline H38 & -2.8503300273 & 8.0679127738 & 15.6861663089 \\
\hline H39 & -2.7721810527 & 10.2410031425 & 14.4912638377 \\
\hline $\mathrm{H} 40$ & -0.6891753851 & 10.9363139591 & 13.3437354931 \\
\hline $\mathrm{H} 41$ & 0.6219762663 & 9.7637168838 & 11.2397396868 \\
\hline $\mathrm{H} 42$ & 2.5734535746 & 10.6663767385 & 10.0198517007 \\
\hline $\mathrm{H} 43$ & 4.7244361474 & 11.0169935392 & 11.2185212431 \\
\hline H44 & 4.8668175242 & 10.5516494510 & 13.6383592274 \\
\hline H45 & 1.8538453785 & 11.6384219684 & 15.6332659472 \\
\hline $\mathrm{H} 46$ & 2.2405236910 & 11.6909840976 & 18.0827115589 \\
\hline $\mathrm{H} 47$ & 3.6673799198 & 9.9687950605 & 19.1478678172 \\
\hline $\mathrm{H} 48$ & 5.0597355499 & 7.8945821966 & 18.9224494747 \\
\hline H49 & 5.8890249925 & 6.0499379092 & 17.4517228143 \\
\hline H50 & 5.0432465141 & 5.9430186062 & 15.0707272779 \\
\hline H51 & 3.1314443743 & 7.1091244197 & 11.2038049371 \\
\hline H52 & 5.2214539051 & 6.9167711958 & 12.3955045674 \\
\hline O49 & 2.8347804164 & 3.8619383668 & 13.6120286211 \\
\hline H53 & 3.6424922682 & 3.5668908681 & 13.1786411446 \\
\hline H54 & 1.8771981085 & 4.3457689581 & 12.5411989548 \\
\hline O52 & 1.2398287435 & 4.7522599093 & 11.8374754378 \\
\hline H55 & 1.2856368393 & 4.1638063624 & 11.0794835151 \\
\hline H56 & 1.9068876007 & 6.1455216906 & 11.6685156966 \\
\hline \multicolumn{4}{|c|}{$6 \mathrm{FP}-\mathrm{Co}(\mathrm{OH})_{2}(\mathrm{O})-2\left(\mathrm{H}_{2} \mathrm{O}\right)-$ Path 3 TS } \\
\hline Col & 3.1798496056 & 7.1238253163 & 13.2323395496 \\
\hline $\mathrm{O} 2$ & 4.8511842706 & 7.1369892636 & 12.2716252514 \\
\hline $\mathrm{O} 3$ & 2.2032492530 & 7.0532994660 & 11.8159517319 \\
\hline O50 & 3.7412644691 & 5.1182156146 & 13.5066632454 \\
\hline N9 & 1.4942002252 & 7.0015090963 & 14.4125026480 \\
\hline N10 & 4.2160044752 & 7.6795927143 & 14.9305880336 \\
\hline $\mathrm{C} 11$ & 1.5068939553 & 5.9270992090 & 15.1545042514 \\
\hline $\mathrm{C} 12$ & 0.3861287370 & 5.4943916368 & 15.9077862647 \\
\hline $\mathrm{C} 13$ & -0.7726735407 & 6.2266338163 & 15.8456668572 \\
\hline $\mathrm{C} 14$ & -0.7988840160 & 7.4016237183 & 15.0764203878 \\
\hline $\mathrm{C} 15$ & 0.3736560699 & 7.7993567468 & 14.3747900028 \\
\hline $\mathrm{C} 16$ & -1.9618859630 & 8.1795461278 & 15.0018586385 \\
\hline $\mathrm{C} 17$ & -1.9788703896 & 9.3267938451 & 14.2798729631 \\
\hline $\mathrm{C} 18$ & -0.8243464310 & 9.7408985236 & 13.6131729037 \\
\hline C19 & 0.3340786136 & 9.0380655034 & 13.6453152274 \\
\hline $\mathrm{C} 20$ & 1.4768357454 & 9.6151589453 & 12.9166023067 \\
\hline $\mathrm{C} 21$ & 1.3780449695 & 9.8911556821 & 11.5568907052 \\
\hline $\mathrm{C} 22$ & 2.4621135640 & 10.3369674558 & 10.8475545026 \\
\hline $\mathrm{C} 23$ & 3.6948359665 & 10.5002581818 & 11.4793897129 \\
\hline $\mathrm{C} 24$ & 3.8210514112 & 10.2467123207 & 12.8435445342 \\
\hline $\mathrm{C} 25$ & 2.7022526688 & 9.8092536257 & 13.5913897117 \\
\hline
\end{tabular}




\begin{tabular}{|c|c|c|c|}
\hline $\mathrm{C} 26$ & 2.8574185051 & 9.6990469685 & 15.0816055856 \\
\hline $\mathrm{C} 27$ & 2.2712663343 & 10.6456694406 & 15.8751943839 \\
\hline $\mathrm{C} 28$ & 2.5420172867 & 10.7322198192 & 17.2458002368 \\
\hline $\mathrm{C} 29$ & 3.4514537134 & 9.9026699365 & 17.8240276288 \\
\hline C30 & 4.0799899230 & 8.8977115567 & 17.0622208326 \\
\hline C31 & 3.7311978363 & 8.7417372479 & 15.6888310745 \\
\hline C32 & 5.0688367351 & 8.0319179462 & 17.5851057029 \\
\hline C33 & 5.6272824087 & 7.0691764887 & 16.7632482651 \\
\hline C34 & 5.1522969490 & 6.8929737904 & 15.4326685166 \\
\hline H35 & 2.4548449133 & 5.3801143153 & 15.1263450485 \\
\hline H36 & 0.4566998272 & 4.5804414690 & 16.4964913464 \\
\hline H37 & -1.6663309681 & 5.9307595581 & 16.3900138499 \\
\hline H38 & -2.8385898080 & 7.8653415300 & 15.5556511898 \\
\hline H39 & -2.8757134020 & 9.9305110199 & 14.2226412975 \\
\hline $\mathrm{H} 40$ & -0.8205905788 & 10.6784644882 & 13.0728184322 \\
\hline $\mathrm{H} 41$ & 0.4602999247 & 9.6544079740 & 11.0450350322 \\
\hline $\mathrm{H} 42$ & 2.3673725599 & 10.5123462736 & 9.7817924638 \\
\hline $\mathrm{H} 43$ & 4.5747545830 & 10.8186812517 & 10.9207904532 \\
\hline H44 & 4.7842288702 & 10.3626092531 & 13.3428950956 \\
\hline H45 & 1.6050291997 & 11.3595805034 & 15.4108449454 \\
\hline $\mathrm{H} 46$ & 2.0609374485 & 11.4996818905 & 17.8333529607 \\
\hline $\mathrm{H} 47$ & 3.7016470111 & 9.9949431617 & 18.8752800144 \\
\hline $\mathrm{H} 48$ & 5.3770315792 & 8.1506031638 & 18.6190293664 \\
\hline H49 & 6.3979960595 & 6.3989386777 & 17.1167529410 \\
\hline H50 & 5.4865584816 & 6.1024371805 & 14.7630018723 \\
\hline H51 & 4.4263596448 & 5.2106801360 & 12.8514382296 \\
\hline H52 & 4.6608717957 & 7.1696049281 & 11.3022382065 \\
\hline H54 & 2.7864522173 & 4.4406309216 & 13.0405911898 \\
\hline O52 & 1.7267919895 & 3.8905372324 & 12.6378219365 \\
\hline H55 & 1.2474838183 & 3.6230760551 & 13.3900279526 \\
\hline H57 & 1.1707896460 & 4.9589714213 & 12.2676116467 \\
\hline O56 & 0.6189848088 & 6.0508044123 & 11.9664520123 \\
\hline $\mathrm{H} 58$ & 0.5929888621 & 6.1010500124 & 11.0659621698 \\
\hline \multicolumn{4}{|c|}{$6 \mathrm{FP}-\mathrm{Co}(\mathrm{OH})_{2}(\mathrm{O})-2\left(\mathrm{H}_{2} \mathrm{O}\right)-$ Path $4 \mathrm{TS}$} \\
\hline Col & 3.1127620722 & 7.3427566987 & 12.9658161124 \\
\hline $\mathrm{O} 2$ & 4.6802151449 & 7.9549086284 & 12.0520151749 \\
\hline $\mathrm{O} 3$ & 2.1074990064 & 7.3563118572 & 11.3746115113 \\
\hline O50 & 3.7322403685 & 5.7225166922 & 12.8291802167 \\
\hline N9 & 1.3581448838 & 7.0699824062 & 14.0844082658 \\
\hline N10 & 3.9433819947 & 7.6359993357 & 14.9544944214 \\
\hline $\mathrm{C} 11$ & 1.2788109192 & 5.8592868110 & 14.6207572216 \\
\hline $\mathrm{C} 12$ & 0.2393301758 & 5.4559709285 & 15.4825456750 \\
\hline $\mathrm{C} 13$ & -0.7771208630 & 6.3323650360 & 15.7559062877 \\
\hline $\mathrm{C} 14$ & -0.7535710995 & 7.6242721349 & 15.1749982770 \\
\hline $\mathrm{C} 15$ & 0.3649437021 & 7.9900860310 & 14.3549401037 \\
\hline $\mathrm{C} 16$ & -1.8041852459 & 8.5485573267 & 15.4089465983 \\
\hline $\mathrm{C} 17$ & -1.7622050725 & 9.8004142903 & 14.8480330671 \\
\hline $\mathrm{C} 18$ & -0.6438863228 & 10.1791093144 & 14.0766844049 \\
\hline C19 & 0.4203310807 & 9.3240884604 & 13.8433401410 \\
\hline $\mathrm{C} 20$ & 1.6012558345 & 9.8700105993 & 13.1198900846 \\
\hline $\mathrm{C} 21$ & 1.4674330740 & 10.3227461779 & 11.7994816405 \\
\hline $\mathrm{C} 22$ & 2.5229629116 & 10.9483133208 & 11.1507210475 \\
\hline $\mathrm{C} 23$ & 3.7415412073 & 11.1243182084 & 11.8119411628 \\
\hline $\mathrm{C} 24$ & 3.8851977349 & 10.6867426695 & 13.1210647550 \\
\hline $\mathrm{C} 25$ & 2.8207161322 & 10.0663489009 & 13.7921755438 \\
\hline
\end{tabular}




\begin{tabular}{|c|c|c|c|}
\hline $\mathrm{C} 26$ & 2.9440036262 & 9.8272262607 & 15.2627089738 \\
\hline $\mathrm{C} 27$ & 2.5000207172 & 10.8091954504 & 16.1305686067 \\
\hline $\mathrm{C} 28$ & 2.6597930409 & 10.6980696804 & 17.5289908168 \\
\hline C29 & 3.3019965661 & 9.6040659262 & 18.0588686433 \\
\hline C30 & 3.7780704925 & 8.5734597673 & 17.2065239019 \\
\hline C31 & 3.5704603078 & 8.6637724869 & 15.7918479223 \\
\hline C32 & 4.4608689796 & 7.4376264254 & 17.7116877277 \\
\hline C33 & 4.8863444486 & 6.4670961345 & 16.8402698446 \\
\hline C34 & 4.5833745128 & 6.5994218299 & 15.4665958595 \\
\hline H35 & 2.0616736610 & 5.1598226108 & 14.3316993805 \\
\hline H36 & 0.2645358871 & 4.4574503244 & 15.9040760866 \\
\hline H37 & -1.6046982598 & 6.0587182140 & 16.4041454348 \\
\hline H38 & -2.6380452135 & 8.2411053286 & 16.0323790697 \\
\hline H39 & -2.5655461915 & 10.5110913168 & 15.0138446409 \\
\hline $\mathrm{H} 40$ & -0.5908585269 & 11.1901708116 & 13.6866000641 \\
\hline $\mathrm{H} 41$ & 0.5234355808 & 10.1708001418 & 11.2887631224 \\
\hline $\mathrm{H} 42$ & 2.3994107998 & 11.3002268859 & 10.1312795008 \\
\hline $\mathrm{H} 43$ & 4.5697677572 & 11.6136726803 & 11.3100033301 \\
\hline H44 & 4.8183345840 & 10.8425207030 & 13.6512801497 \\
\hline H45 & 2.0239231154 & 11.6939077926 & 15.7200209791 \\
\hline H46 & 2.2876132620 & 11.4883293453 & 18.1739824165 \\
\hline H47 & 3.4560718586 & 9.5112719011 & 19.1301834641 \\
\hline $\mathrm{H} 48$ & 4.6363360764 & 7.3552458673 & 18.7809940626 \\
\hline H49 & 5.4202512556 & 5.5882603794 & 17.1840494643 \\
\hline $\mathrm{H} 50$ & 4.8220708864 & 5.8162229775 & 14.7562069254 \\
\hline H51 & 2.7714169855 & 7.6479326234 & 10.7363390428 \\
\hline H52 & 5.1606237142 & 7.1267977743 & 11.9253577691 \\
\hline $\mathrm{O} 49$ & 3.0772935608 & 3.9011589342 & 13.1961151895 \\
\hline H54 & 1.4840639476 & 5.7706449843 & 11.3544189244 \\
\hline O52 & 1.1764371575 & 4.8296597939 & 11.4028790221 \\
\hline H55 & 1.8645414504 & 4.4730719053 & 12.0008805226 \\
\hline H53 & 3.9254897993 & 3.8016522125 & 13.6422784402 \\
\hline \multicolumn{4}{|c|}{$5 \mathrm{FP}-\mathrm{Co}(\mathrm{OH})_{2}\left(\mathrm{H}_{2} \mathrm{O}\right)$} \\
\hline Co1 & 4.4490171123 & 13.3355698010 & 3.1688514492 \\
\hline $\mathrm{O} 2$ & 3.8110433666 & 11.6253958512 & 3.6985368077 \\
\hline $\mathrm{O} 3$ & 5.8847645961 & 13.1433447365 & 4.4027958042 \\
\hline $\mathrm{O} 4$ & 3.4299741848 & 13.7788543365 & 5.3150740346 \\
\hline N7 & 5.0388696349 & 15.1695725403 & 2.6707464012 \\
\hline N8 & 6.2161413651 & 14.5367370578 & 0.6600875740 \\
\hline N9 & 3.9961546044 & 12.8937288106 & -0.0711945132 \\
\hline N10 & 2.8947409119 & 13.6042974120 & 1.9568872285 \\
\hline $\mathrm{C} 12$ & 4.6419693364 & 16.2212060270 & 3.4058553375 \\
\hline H13 & 4.1043731124 & 15.9660554308 & 4.3107104965 \\
\hline C14 & 4.8849008824 & 17.5462309553 & 3.0288559674 \\
\hline H15 & 4.5323708009 & 18.3423029158 & 3.6718305786 \\
\hline $\mathrm{C} 16$ & 5.5615169645 & 17.8315383767 & 1.8479611226 \\
\hline H17 & 5.7477402957 & 18.8553498892 & 1.5448278272 \\
\hline $\mathrm{C} 18$ & 5.9979175565 & 16.7569856293 & 1.0686328735 \\
\hline C19 & 6.7172344262 & 16.5854674483 & -0.1602493179 \\
\hline $\mathrm{H} 20$ & 7.0963972285 & 17.3612409856 & -0.8057468039 \\
\hline $\mathrm{C} 21$ & 6.8356171733 & 15.2399577197 & -0.3680816248 \\
\hline $\mathrm{H} 22$ & 7.3118494108 & 14.6954263271 & -1.1674132917 \\
\hline $\mathrm{C} 23$ & 5.7042539227 & 15.4549995017 & 1.5489441847 \\
\hline $\mathrm{C} 24$ & 6.2591912815 & 13.1225773304 & 0.8637217120 \\
\hline $\mathrm{C} 25$ & 7.3762567817 & 12.5486687516 & 1.4649507192 \\
\hline
\end{tabular}




\begin{tabular}{|c|c|c|c|}
\hline $\mathrm{H} 26$ & 8.1917876350 & 13.1910261041 & 1.7712247145 \\
\hline $\mathrm{C} 27$ & 7.4097797085 & 11.1815927236 & 1.7098655380 \\
\hline $\mathrm{H} 28$ & 8.2651187475 & 10.7484643719 & 2.2125291312 \\
\hline $\mathrm{C} 29$ & 6.3296084940 & 10.3813424013 & 1.3518120402 \\
\hline H30 & 6.3394706689 & 9.3221143472 & 1.5749636472 \\
\hline C 31 & 5.2128030928 & 10.9461336602 & 0.7486581692 \\
\hline H32 & 4.3477038346 & 10.3446286878 & 0.5012587871 \\
\hline C33 & 5.1702778126 & 12.3160040989 & 0.5033450598 \\
\hline $\mathrm{C} 34$ & 3.6389346426 & 12.8676299953 & -1.4154816593 \\
\hline H35 & 4.3357479083 & 12.4761490275 & -2.1389549315 \\
\hline C36 & 2.3831613587 & 13.3801054627 & -1.5821444826 \\
\hline H37 & 1.8630387107 & 13.4878611024 & -2.5202876985 \\
\hline C38 & 1.9031265919 & 13.7418948472 & -0.2799844742 \\
\hline C39 & 0.7330020531 & 14.2867823810 & 0.2549801197 \\
\hline $\mathrm{H} 40$ & -0.1007585579 & 14.5580201485 & -0.3822226133 \\
\hline C41 & 0.6686108814 & 14.4643983073 & 1.6326677730 \\
\hline H42 & -0.2176192135 & 14.8766350615 & 2.0975063085 \\
\hline $\mathrm{C} 43$ & 1.7535339331 & 14.1178078387 & 2.4446680743 \\
\hline H44 & 1.7401522802 & 14.2557674078 & 3.5187731095 \\
\hline C45 & 2.9389557045 & 13.4219199946 & 0.6349853839 \\
\hline $\mathrm{H} 46$ & 3.0923771842 & 11.3321034432 & 3.1291264338 \\
\hline H47 & 6.5369707559 & 13.8421583954 & 4.2887032765 \\
\hline $\mathrm{H} 45$ & 3.2122114546 & 12.8466938619 & 5.1000075880 \\
\hline $\mathrm{H} 48$ & 4.3990319538 & 13.6813489424 & 5.4575662550 \\
\hline \multicolumn{4}{|c|}{$5 \mathrm{FP}-\mathrm{Co}(\mathrm{OH})_{3}$} \\
\hline Co1 & 4.3865931143 & 13.3152428171 & 3.2427124106 \\
\hline $\mathrm{O} 2$ & 3.8814168709 & 11.5505327254 & 3.4454691431 \\
\hline $\mathrm{O} 3$ & 5.9208405169 & 12.9947725402 & 4.1610513693 \\
\hline $\mathrm{O} 4$ & 3.3944013474 & 13.9213790076 & 4.6660955091 \\
\hline N7 & 5.0426276274 & 15.1873190451 & 2.7447786461 \\
\hline N8 & 6.1716254384 & 14.5491818826 & 0.7000590530 \\
\hline N9 & 3.9702580341 & 12.8444939553 & 0.0220695139 \\
\hline N10 & 2.8346126892 & 13.6711283016 & 1.9884899099 \\
\hline $\mathrm{C} 12$ & 4.6986798234 & 16.2378109465 & 3.5009742552 \\
\hline H13 & 4.1519743978 & 15.9721129484 & 4.3965048665 \\
\hline $\mathrm{C} 14$ & 4.9863594148 & 17.5600297199 & 3.1446047304 \\
\hline H15 & 4.6730443552 & 18.3563304602 & 3.8076156478 \\
\hline $\mathrm{C} 16$ & 5.6503159783 & 17.8416976532 & 1.9574063288 \\
\hline H17 & 5.8683432358 & 18.8627572089 & 1.6654593690 \\
\hline $\mathrm{C} 18$ & 6.0334866215 & 16.7649920093 & 1.1547585922 \\
\hline C19 & 6.7289320417 & 16.5953977355 & -0.0882124916 \\
\hline $\mathrm{H} 20$ & 7.1190371752 & 17.3723975545 & -0.7258403601 \\
\hline $\mathrm{C} 21$ & 6.8034783834 & 15.2524690860 & -0.3218692454 \\
\hline $\mathrm{H} 22$ & 7.2510498748 & 14.7075648623 & -1.1372608494 \\
\hline $\mathrm{C} 23$ & 5.7037774272 & 15.4648492785 & 1.6183218275 \\
\hline $\mathrm{C} 24$ & 6.2482890965 & 13.1389536134 & 0.9060577471 \\
\hline $\mathrm{C} 25$ & 7.4108912101 & 12.5915360722 & 1.4428054585 \\
\hline H26 & 8.2248514365 & 13.2539512664 & 1.7053334847 \\
\hline $\mathrm{C} 27$ & 7.4863054975 & 11.2279283751 & 1.6872961478 \\
\hline $\mathrm{H} 28$ & 8.3838083465 & 10.8120217050 & 2.1284088077 \\
\hline $\mathrm{C} 29$ & 6.3961504605 & 10.4052512801 & 1.4106120639 \\
\hline $\mathrm{H} 30$ & 6.4400781136 & 9.3473929353 & 1.6377936913 \\
\hline $\mathrm{C} 31$ & 5.2336943133 & 10.9431647819 & 0.8777873039 \\
\hline H32 & 4.3623522536 & 10.3291840647 & 0.6982966715 \\
\hline C33 & 5.1558535022 & 12.3103521039 & 0.6142154404 \\
\hline
\end{tabular}




\begin{tabular}{|c|c|c|c|}
\hline $\mathrm{C} 34$ & 3.6332697534 & 12.7429598292 & -1.3250617878 \\
\hline H35 & 4.3417467254 & 12.3114960584 & -2.0136326028 \\
\hline C36 & 2.3813896376 & 13.2446432093 & -1.5392040364 \\
\hline H37 & 1.8742537464 & 13.2984342623 & -2.4890788867 \\
\hline C38 & 1.8835275302 & 13.6797545785 & -0.2668206164 \\
\hline C39 & 0.7071548003 & 14.2580891653 & 0.2148702692 \\
\hline $\mathrm{H} 40$ & -0.1148965451 & 14.4919552697 & -0.4519781257 \\
\hline C41 & 0.6220505215 & 14.5185444455 & 1.5771252626 \\
\hline $\mathrm{H} 42$ & -0.2700006344 & 14.9601172875 & 2.0025346525 \\
\hline $\mathrm{C} 43$ & 1.6925062092 & 14.2172984187 & 2.4266044092 \\
\hline H44 & 1.6701355286 & 14.4033029549 & 3.4927625851 \\
\hline $\mathrm{C} 45$ & 2.9040914930 & 13.4106512292 & 0.6820413180 \\
\hline $\mathrm{H} 46$ & 3.0928180469 & 11.5632122230 & 3.9993265769 \\
\hline $\mathrm{H} 47$ & 5.7968601953 & 12.0809228208 & 4.4516147544 \\
\hline $\mathrm{H} 45$ & 3.9361839259 & 13.6663214153 & 5.4235139853 \\
\hline \multicolumn{4}{|c|}{$5 \mathrm{FP}-\mathrm{Co}(\mathrm{OH})_{2}(\mathrm{O})$} \\
\hline $\mathrm{Co} 1$ & 4.3449498319 & 13.3287672083 & 3.2938244143 \\
\hline $\mathrm{O} 2$ & 3.7658694630 & 11.5901570472 & 3.4764495744 \\
\hline $\mathrm{O} 3$ & 5.9190682055 & 12.9089517769 & 4.0368758457 \\
\hline $\mathrm{O} 4$ & 3.3920011896 & 13.9361651923 & 4.5899783917 \\
\hline N7 & 5.0819583072 & 15.1911445592 & 2.7743927401 \\
\hline N8 & 6.1193796469 & 14.5547012455 & 0.6744325653 \\
\hline N9 & 3.9626852496 & 12.7819135449 & 0.0428802220 \\
\hline N10 & 2.8351035425 & 13.7142530973 & 1.9694323995 \\
\hline $\mathrm{C} 12$ & 4.8032368005 & 16.2369559920 & 3.5630513960 \\
\hline H13 & 4.2986201877 & 15.9711235076 & 4.4833489399 \\
\hline $\mathrm{C} 14$ & 5.1019801142 & 17.5576310946 & 3.2109830801 \\
\hline H15 & 4.8424906477 & 18.3510139938 & 3.9001427801 \\
\hline $\mathrm{C} 16$ & 5.7067377557 & 17.8411185779 & 1.9930865377 \\
\hline H17 & 5.9280012868 & 18.8614664448 & 1.7011799793 \\
\hline $\mathrm{C} 18$ & 6.0300291606 & 16.7678055747 & 1.1603420788 \\
\hline C19 & 6.6673405453 & 16.6046795934 & -0.1142550671 \\
\hline $\mathrm{H} 20$ & 7.0393624779 & 17.3845728282 & -0.7590545274 \\
\hline $\mathrm{C} 21$ & 6.7167320385 & 15.2642779941 & -0.3646690558 \\
\hline $\mathrm{H} 22$ & 7.1278974372 & 14.7235613924 & -1.2017775781 \\
\hline $\mathrm{C} 23$ & 5.7013612979 & 15.4668206428 & 1.6237383035 \\
\hline $\mathrm{C} 24$ & 6.2422497052 & 13.1470793100 & 0.8838251415 \\
\hline $\mathrm{C} 25$ & 7.4311890111 & 12.6367769639 & 1.3956403416 \\
\hline H26 & 8.2390004031 & 13.3211875396 & 1.6190312416 \\
\hline $\mathrm{C} 27$ & 7.5429315003 & 11.2807656529 & 1.6694444507 \\
\hline $\mathrm{H} 28$ & 8.4624107976 & 10.8937584568 & 2.0912937088 \\
\hline $\mathrm{C} 29$ & 6.4614353983 & 10.4304976003 & 1.4498296126 \\
\hline H30 & 6.5338596872 & 9.3798334198 & 1.7020070465 \\
\hline C31 & 5.2736598949 & 10.9311681678 & 0.9354630344 \\
\hline $\mathrm{H} 32$ & 4.4103560330 & 10.2961502916 & 0.7938255033 \\
\hline C33 & 5.1634694714 & 12.2884771096 & 0.6385131415 \\
\hline C34 & 3.6305910995 & 12.6361532617 & -1.3013023104 \\
\hline H35 & 4.3336598197 & 12.1627384415 & -1.9674220369 \\
\hline C36 & 2.3915143419 & 13.1552694239 & -1.5426499854 \\
\hline H37 & 1.8879732519 & 13.1795460939 & -2.4955320921 \\
\hline C38 & 1.8993379404 & 13.6528842460 & -0.2921721891 \\
\hline C39 & 0.7325431376 & 14.2750973209 & 0.1531210593 \\
\hline $\mathrm{H} 40$ & -0.0790052515 & 14.4970225856 & -0.5302892413 \\
\hline $\mathrm{C} 41$ & 0.6431533713 & 14.5960754964 & 1.5019786731 \\
\hline $\mathrm{H} 42$ & -0.2413359768 & 15.0738322622 & 1.9029939275 \\
\hline
\end{tabular}




$\begin{array}{llll}\text { C43 } & 1.6996788190 & 14.3044455886 & 2.3692550701 \\ \text { H44 } & 1.6585248680 & 14.5346034139 & 3.4260318545 \\ \text { C45 } & 2.9104638228 & 13.3997721736 & 0.6743871597 \\ \text { H46 } & 3.0839538282 & 11.6281081341 & 4.1580460357 \\ \text { H47 } & 5.7630362235 & 12.0246612323 & 4.3963582789\end{array}$

\section{FP-Co $(\mathrm{OH})_{2}(\mathrm{O})$ - $2\left(\mathrm{H}_{2} \mathrm{O}\right)$ - Path 1 TS}

\begin{tabular}{|c|c|c|c|}
\hline Co1 & 4.4312226730 & 13.4219934042 & 3.3323499250 \\
\hline $\mathrm{O} 2$ & 3.5731248685 & 12.1308619956 & 4.5152049547 \\
\hline $\mathrm{O} 3$ & 6.1089977990 & 13.1194038169 & 3.9475608716 \\
\hline $\mathrm{O} 4$ & 3.4270487050 & 13.8734717492 & 4.9417907296 \\
\hline N7 & 5.0093136840 & 15.1726551529 & 2.5662449203 \\
\hline N8 & 6.3752916329 & 14.4567959463 & 0.6932555784 \\
\hline N9 & 3.9539515273 & 13.0675656730 & 0.1017977253 \\
\hline N10 & 2.7762472288 & 13.5119017720 & 2.1591193116 \\
\hline $\mathrm{C} 12$ & 4.5724187886 & 16.2558796520 & 3.2287017023 \\
\hline H13 & 3.9505937937 & 16.0466446808 & 4.0903333626 \\
\hline C14 & 4.8952956009 & 17.5619363064 & 2.8511447659 \\
\hline H15 & 4.5037163677 & 18.3836605902 & 3.4363469803 \\
\hline $\mathrm{C} 16$ & 5.7112408107 & 17.7930452030 & 1.7507469595 \\
\hline H17 & 5.9738771474 & 18.8008622475 & 1.4505621989 \\
\hline $\mathrm{C} 18$ & 6.1900924573 & 16.6855608324 & 1.0494086408 \\
\hline C19 & 7.0423501326 & 16.4736848549 & -0.0836053631 \\
\hline $\mathrm{H} 20$ & 7.5212817915 & 17.2282914008 & -0.6863056632 \\
\hline $\mathrm{C} 21$ & 7.1362485359 & 15.1239017395 & -0.2623502340 \\
\hline $\mathrm{H} 22$ & 7.6768149426 & 14.5507135326 & -0.9982186273 \\
\hline $\mathrm{C} 23$ & 5.7957726420 & 15.4011562706 & 1.5116739873 \\
\hline $\mathrm{C} 24$ & 6.2978553648 & 13.0468869125 & 0.8608604870 \\
\hline $\mathrm{C} 25$ & 7.4039598641 & 12.3442824547 & 1.3277614092 \\
\hline H26 & 8.3103878573 & 12.8898998911 & 1.5530814233 \\
\hline $\mathrm{C} 27$ & 7.3089710121 & 10.9805982233 & 1.5693772190 \\
\hline $\mathrm{H} 28$ & 8.1670913171 & 10.4447574471 & 1.9558324928 \\
\hline $\mathrm{C} 29$ & 6.1019163107 & 10.3149251334 & 1.3672645751 \\
\hline $\mathrm{H} 30$ & 6.0199512439 & 9.2564716032 & 1.5828102750 \\
\hline $\mathrm{C} 31$ & 4.9949668976 & 11.0107250015 & 0.8970929936 \\
\hline H32 & 4.0468580554 & 10.5141610982 & 0.7322270201 \\
\hline C33 & 5.0904591647 & 12.3779536109 & 0.6307628994 \\
\hline C34 & 3.6120536631 & 13.1273883056 & -1.2494044438 \\
\hline H35 & 4.3438265932 & 12.8510962413 & -1.9912111004 \\
\hline C36 & 2.3211806496 & 13.5481204973 & -1.3921945279 \\
\hline H37 & 1.8052983777 & 13.6986596437 & -2.3269196857 \\
\hline C38 & 1.7939370460 & 13.7441806459 & -0.0707449238 \\
\hline C39 & 0.5702264822 & 14.1155591758 & 0.4933826436 \\
\hline $\mathrm{H} 40$ & -0.2800341234 & 14.3683054244 & -0.1298412732 \\
\hline C41 & 0.4744664564 & 14.1403497944 & 1.8805086926 \\
\hline $\mathrm{H} 42$ & -0.4557523575 & 14.4074794950 & 2.3653209715 \\
\hline $\mathrm{C} 43$ & 1.5850438771 & 13.8411497229 & 2.6786400864 \\
\hline $\mathrm{H} 44$ & 1.5810624903 & 13.8838775874 & 3.7612039689 \\
\hline $\mathrm{C} 45$ & 2.8437156310 & 13.4392054255 & 0.8297389048 \\
\hline $\mathrm{H} 46$ & 3.6618651571 & 11.1875060723 & 4.2874381144 \\
\hline $\mathrm{H} 47$ & 5.9994042463 & 12.5325821217 & 4.7056006683 \\
\hline H54 & 3.6784066971 & 16.1267001107 & 6.4530909817 \\
\hline O52 & 2.8604407609 & 16.4246172693 & 6.0140056785 \\
\hline H55 & 2.4875622383 & 15.5773647290 & 5.7348839373 \\
\hline $\mathrm{H} 48$ & 4.8160101734 & 14.3470173721 & 7.6568094798 \\
\hline O49 & 5.0554830978 & 14.8150490296 & 6.8514345371 \\
\hline
\end{tabular}


$\begin{array}{llll}\mathrm{H} 50 & 4.6023894193 & 14.3041708876 & 6.1360133664\end{array}$

\begin{tabular}{|c|c|c|c|}
\hline \multicolumn{4}{|c|}{$5 \mathrm{FP}-\mathrm{Co}(\mathrm{OH})_{2}(\mathrm{O})-2\left(\mathrm{H}_{2} \mathrm{O}\right)-$ Path 2 TS1 } \\
\hline Co1 & 4.4592379193 & 13.4081437705 & 3.2859849028 \\
\hline $\mathrm{O} 2$ & 4.1396599735 & 11.5987744939 & 3.6673620225 \\
\hline $\mathrm{O} 3$ & 6.1384124867 & 13.1756564704 & 4.0331294616 \\
\hline $\mathrm{O} 4$ & 3.7963447433 & 14.0900040433 & 4.7771098112 \\
\hline N7 & 4.9526093316 & 15.2289284746 & 2.6090910317 \\
\hline N8 & 6.3984649554 & 14.4411682195 & 0.8307271890 \\
\hline N9 & 3.9712843163 & 13.0979970186 & 0.1101583644 \\
\hline N10 & 2.7965532096 & 13.5922539283 & 2.1701494341 \\
\hline $\mathrm{C} 12$ & 4.4504480678 & 16.3322254735 & 3.1837921755 \\
\hline H13 & 3.7870524819 & 16.1322509356 & 4.0155742321 \\
\hline $\mathrm{C} 14$ & 4.7709888240 & 17.6222069351 & 2.7526740333 \\
\hline H15 & 4.3282002678 & 18.4648015027 & 3.2674982949 \\
\hline $\mathrm{C} 16$ & 5.6430032604 & 17.8133536308 & 1.6881174242 \\
\hline H17 & 5.9023268692 & 18.8096318165 & 1.3488838266 \\
\hline $\mathrm{C} 18$ & 6.1743474422 & 16.6816883714 & 1.0679368219 \\
\hline $\mathrm{C} 19$ & 7.0726985745 & 16.4263659930 & -0.0197860844 \\
\hline $\mathrm{H} 20$ & 7.5671953349 & 17.1572998282 & -0.6388606498 \\
\hline $\mathrm{C} 21$ & 7.1843063960 & 15.0711471195 & -0.1320327844 \\
\hline $\mathrm{H} 22$ & 7.7517904884 & 14.4690889006 & -0.8230830918 \\
\hline $\mathrm{C} 23$ & 5.7801876439 & 15.4157527682 & 1.5763498616 \\
\hline $\mathrm{C} 24$ & 6.2734949301 & 13.0310585742 & 0.9898312971 \\
\hline $\mathrm{C} 25$ & 7.3509635837 & 12.2975472228 & 1.4833252576 \\
\hline H26 & 8.2384684898 & 12.8280712488 & 1.7990579649 \\
\hline $\mathrm{C} 27$ & 7.2504231206 & 10.9201541060 & 1.6124182213 \\
\hline $\mathrm{H} 28$ & 8.0849912039 & 10.3589553348 & 2.0145478593 \\
\hline $\mathrm{C} 29$ & 6.0686985074 & 10.2659086358 & 1.2660865626 \\
\hline H30 & 5.9810840156 & 9.1940833713 & 1.3942154124 \\
\hline C31 & 4.9888468021 & 10.9907572687 & 0.7854223441 \\
\hline H32 & 4.0519707785 & 10.5074968667 & 0.5409324286 \\
\hline $\mathrm{C} 33$ & 5.0848765929 & 12.3751397824 & 0.6381855924 \\
\hline C34 & 3.6459150096 & 13.1885492864 & -1.2441029297 \\
\hline H35 & 4.3704610415 & 12.8828881931 & -1.9813125982 \\
\hline C36 & 2.3824654325 & 13.6811882129 & -1.3907105971 \\
\hline H37 & 1.8811651020 & 13.8706577925 & -2.3262990507 \\
\hline C38 & 1.8591398370 & 13.8981085902 & -0.0712821469 \\
\hline C39 & 0.6595680606 & 14.3589171917 & 0.4789464255 \\
\hline $\mathrm{H} 40$ & -0.1634731134 & 14.6687062177 & -0.1552287140 \\
\hline $\mathrm{C} 41$ & 0.5562556267 & 14.3997204824 & 1.8619557218 \\
\hline $\mathrm{H} 42$ & -0.3543568503 & 14.7323740031 & 2.3429454584 \\
\hline C43 & 1.6301712047 & 14.0234076646 & 2.6792091863 \\
\hline $\mathrm{H} 44$ & 1.5695096605 & 14.0513613254 & 3.7695459893 \\
\hline $\mathrm{C} 45$ & 2.8766766588 & 13.5191248458 & 0.8355479469 \\
\hline $\mathrm{H} 46$ & 4.8421630927 & 11.4350770024 & 4.3131230360 \\
\hline $\mathrm{H} 47$ & 6.1991327156 & 13.7307732110 & 4.8182737003 \\
\hline $\mathrm{O} 45$ & 1.8364312827 & 11.1888554381 & 4.5971940937 \\
\hline $\mathrm{H} 48$ & 1.8646057853 & 10.5222251738 & 5.2904715474 \\
\hline $\mathrm{H} 49$ & 3.0054107889 & 11.3439661154 & 4.1533162286 \\
\hline $\mathrm{O} 48$ & 1.5110338669 & 13.4736265439 & 5.6010378449 \\
\hline $\mathrm{H} 50$ & 1.6410406332 & 12.3050438138 & 5.1481442483 \\
\hline H51 & 2.3640493270 & 13.6471964400 & 6.0178481996 \\
\hline
\end{tabular}

5FP-Co $(\mathrm{OH})_{2}(\mathrm{O})$ - 2( $\left.\mathrm{H}_{2} \mathrm{O}\right)$ - Path 2 Intermediate $\begin{array}{llll}\text { Co1 } & 4.4223322334 & 13.4016132487 & 3.2775500482\end{array}$ 


\begin{tabular}{|c|c|c|c|}
\hline $\mathrm{O} 2$ & 4.0947429945 & 11.5272061949 & 3.6850906458 \\
\hline $\mathrm{O} 3$ & 6.0648904827 & 13.0905151994 & 4.0858065227 \\
\hline $\mathrm{O} 4$ & 3.7001244489 & 14.0587791645 & 4.7808320432 \\
\hline N7 & 4.9557234267 & 15.2088960738 & 2.6450229990 \\
\hline N8 & 6.3582182873 & 14.4441863575 & 0.8220628399 \\
\hline N9 & 3.9547294017 & 13.0618801918 & 0.0866890343 \\
\hline N10 & 2.7869884539 & 13.6198111857 & 2.1353732360 \\
\hline $\mathrm{C} 12$ & 4.4755411030 & 16.3034728696 & 3.2554025119 \\
\hline H13 & 3.8241612352 & 16.0834260261 & 4.0937205873 \\
\hline $\mathrm{C} 14$ & 4.7989257745 & 17.5994690013 & 2.8423973787 \\
\hline H15 & 4.3753285698 & 18.4355289297 & 3.3837516150 \\
\hline $\mathrm{C} 16$ & 5.6471451613 & 17.8057416685 & 1.7608041828 \\
\hline H17 & 5.9055537203 & 18.8068722619 & 1.4347432622 \\
\hline $\mathrm{C} 18$ & 6.1558743055 & 16.6821314142 & 1.1066739005 \\
\hline C19 & 7.0292789449 & 16.4406031767 & -0.0053522718 \\
\hline $\mathrm{H} 20$ & 7.5153637663 & 17.1788341079 & -0.6227622641 \\
\hline $\mathrm{C} 21$ & 7.1299508638 & 15.0861186661 & -0.1444831937 \\
\hline $\mathrm{H} 22$ & 7.6810091949 & 14.4942890673 & -0.8574751166 \\
\hline $\mathrm{C} 23$ & 5.7635188844 & 15.4101192096 & 1.5995898256 \\
\hline $\mathrm{C} 24$ & 6.2522275926 & 13.0325049123 & 0.9835520817 \\
\hline $\mathrm{C} 25$ & 7.3360878994 & 12.3169511713 & 1.4892737128 \\
\hline H26 & 8.2149279288 & 12.8610304978 & 1.8069675713 \\
\hline $\mathrm{C} 27$ & 7.2550858724 & 10.9379477151 & 1.6261316216 \\
\hline $\mathrm{H} 28$ & 8.0948599709 & 10.3912480671 & 2.0373162163 \\
\hline $\mathrm{C} 29$ & 6.0852215367 & 10.2652104452 & 1.2748392898 \\
\hline $\mathrm{H} 30$ & 6.0120454826 & 9.1925233362 & 1.4064711733 \\
\hline C31 & 4.9972327640 & 10.9730419757 & 0.7829079813 \\
\hline H32 & 4.0699309417 & 10.4737117550 & 0.5327746817 \\
\hline C33 & 5.0738163392 & 12.3582764827 & 0.6287507728 \\
\hline C34 & 3.6350062881 & 13.1366287096 & -1.2696373822 \\
\hline H35 & 4.3540169395 & 12.8043468851 & -2.0008703667 \\
\hline C36 & 2.3825293491 & 13.6559234251 & -1.4279450907 \\
\hline H37 & 1.8884874032 & 13.8393433097 & -2.3686984838 \\
\hline C38 & 1.8625685999 & 13.9096758132 & -0.1135074024 \\
\hline C39 & 0.6743385739 & 14.4120283696 & 0.4259635361 \\
\hline $\mathrm{H} 40$ & -0.1401455063 & 14.7291827591 & -0.2157136886 \\
\hline $\mathrm{C} 41$ & 0.5719283174 & 14.4883638705 & 1.8083438821 \\
\hline $\mathrm{H} 42$ & -0.3283435723 & 14.8605802448 & 2.2800636045 \\
\hline $\mathrm{C} 43$ & 1.6352990329 & 14.0998609980 & 2.6348989330 \\
\hline H44 & 1.5939877691 & 14.1700216203 & 3.7226226376 \\
\hline $\mathrm{C} 45$ & 2.8689397122 & 13.5225106576 & 0.8025333225 \\
\hline $\mathrm{H} 46$ & 4.8396673175 & 11.4431246416 & 4.3057816369 \\
\hline $\mathrm{H} 47$ & 6.0692662017 & 13.5803498271 & 4.9165248681 \\
\hline $\mathrm{O} 45$ & 1.8404871953 & 11.2398797397 & 4.8466621925 \\
\hline $\mathrm{H} 48$ & 1.6675251395 & 10.6311266088 & 5.5817835847 \\
\hline $\mathrm{H} 49$ & 3.2235895048 & 11.3541738723 & 4.1846622219 \\
\hline $\mathrm{O} 48$ & 1.6181386390 & 13.6474090097 & 5.5433909453 \\
\hline H50 & 1.7206051066 & 12.1596378798 & 5.1927451172 \\
\hline H51 & 2.1904101853 & 13.9235155766 & 6.2662101423 \\
\hline \multicolumn{4}{|c|}{$5 \mathrm{FP}-\mathrm{Co}(\mathrm{OH})_{2}(\mathrm{O})-2\left(\mathrm{H}_{2} \mathrm{O}\right)-$ Path 2 TS2 } \\
\hline Co1 & 4.3220140313 & 13.3494205825 & 3.2333519730 \\
\hline $\mathrm{O} 2$ & 4.0505788531 & 11.4068658216 & 3.6487537116 \\
\hline $\mathrm{O} 3$ & 5.9500748210 & 13.1733781107 & 4.1919431411 \\
\hline $\mathrm{O} 4$ & 3.4888133973 & 13.9775399846 & 4.7173928328 \\
\hline N7 & 4.7966789737 & 15.1323673866 & 2.6103753172 \\
\hline
\end{tabular}

S18 


\begin{tabular}{|c|c|c|c|}
\hline N8 & 6.4650604064 & 14.3818074757 & 0.9936930867 \\
\hline N9 & 4.0606959645 & 13.2107239161 & -0.0038882353 \\
\hline N10 & 2.7472270907 & 13.5403373955 & 2.0090802795 \\
\hline $\mathrm{C} 12$ & 4.1632105097 & 16.2304972160 & 3.0911973794 \\
\hline H13 & 3.4074578794 & 16.0071828930 & 3.8473016825 \\
\hline C14 & 4.4708977567 & 17.5326365046 & 2.6629865243 \\
\hline H15 & 3.9170150897 & 18.3662567648 & 3.1028662548 \\
\hline $\mathrm{C} 16$ & 5.4641477753 & 17.7538360713 & 1.7004789917 \\
\hline H17 & 5.7157512907 & 18.7657580956 & 1.3668831504 \\
\hline $\mathrm{C} 18$ & 6.1193750135 & 16.6291603322 & 1.1684860315 \\
\hline C19 & 7.1397354963 & 16.3988896878 & 0.1790466423 \\
\hline $\mathrm{H} 20$ & 7.6689322129 & 17.1545607189 & -0.4010408620 \\
\hline $\mathrm{C} 21$ & 7.3205780413 & 15.0354478182 & 0.0920729393 \\
\hline $\mathrm{H} 22$ & 7.9754357259 & 14.4409244436 & -0.5448252414 \\
\hline $\mathrm{C} 23$ & 5.7276702111 & 15.3423828990 & 1.6577559169 \\
\hline C24 & 6.2787265708 & 12.9651020443 & 1.0643777427 \\
\hline $\mathrm{C} 25$ & 7.3006244281 & 12.1397046268 & 1.5572769040 \\
\hline H26 & 8.2126551602 & 12.5994926272 & 1.9467621604 \\
\hline $\mathrm{C} 27$ & 7.1339940619 & 10.7492486561 & 1.5482398431 \\
\hline $\mathrm{H} 28$ & 7.9338252521 & 10.1073686355 & 1.9289274556 \\
\hline $\mathrm{C} 29$ & 5.9488004816 & 10.1756256305 & 1.0559793758 \\
\hline $\mathrm{H} 30$ & 5.8228146970 & 9.0889100174 & 1.0497886581 \\
\hline C31 & 4.9261456377 & 10.9936035007 & 0.5641991050 \\
\hline H32 & 3.9975868085 & 10.5705379909 & 0.1686923639 \\
\hline C33 & 5.0902718291 & 12.3879066392 & 0.5616951502 \\
\hline C34 & 3.8740810086 & 13.4813887892 & -1.3688791598 \\
\hline H35 & 4.6606806158 & 13.2231692369 & -2.0779647263 \\
\hline C36 & 2.6381359899 & 14.0629840875 & -1.5626464256 \\
\hline H37 & 2.2306704471 & 14.3958699639 & -2.5178345126 \\
\hline C 38 & 1.9998261430 & 14.1457361425 & -0.2721674735 \\
\hline C39 & 0.7673894764 & 14.6008237232 & 0.2321615296 \\
\hline $\mathrm{H} 40$ & 0.0079947718 & 15.0316539306 & -0.4290097267 \\
\hline $\mathrm{C} 41$ & 0.5499239000 & 14.4839366619 & 1.6119008790 \\
\hline $\mathrm{H} 42$ & -0.3936665610 & 14.8138017495 & 2.0552402954 \\
\hline $\mathrm{C} 43$ & 1.5467126270 & 13.9747933504 & 2.4678359909 \\
\hline H44 & 1.4382193605 & 13.9393097994 & 3.5568174021 \\
\hline $\mathrm{C} 45$ & 2.9239363703 & 13.6055048199 & 0.6734683944 \\
\hline H46 & 4.9870818846 & 11.1872675426 & 3.8645190001 \\
\hline $\mathrm{H} 47$ & 5.6270120750 & 13.4116631669 & 5.0864784506 \\
\hline $\mathrm{O} 45$ & 3.0503486729 & 11.3179254270 & 6.0364566805 \\
\hline $\mathrm{H} 48$ & 3.8256306649 & 11.6210290188 & 6.5471649084 \\
\hline $\mathrm{H} 49$ & 3.6092249587 & 11.3568418297 & 4.5958988286 \\
\hline $\mathrm{O} 48$ & 1.7851358860 & 13.4703452645 & 5.5895519333 \\
\hline H50 & 2.5007937298 & 12.2200564708 & 5.9001091748 \\
\hline H51 & 2.0157711135 & 14.0692234455 & 6.3271712856 \\
\hline \multicolumn{4}{|c|}{$5 \mathrm{FP}-\mathrm{Co}(\mathrm{OH})_{2}(\mathrm{O})-2\left(\mathrm{H}_{2} \mathrm{O}\right)-$ Path $3 \mathrm{TS}$} \\
\hline $\mathrm{Co} 1$ & 4.4911785543 & 13.4319540881 & 3.3278791636 \\
\hline $\mathrm{O} 2$ & 4.1104452688 & 11.5775152469 & 3.4877010963 \\
\hline $\mathrm{O} 3$ & 6.0512584882 & 13.0935955433 & 4.2647983809 \\
\hline $\mathrm{O} 4$ & 3.6301694608 & 14.1175238267 & 4.7543673622 \\
\hline N7 & 5.0779398794 & 15.2585930554 & 2.8204461933 \\
\hline N8 & 6.1231724203 & 14.4937826682 & 0.7801968645 \\
\hline N9 & 3.9158336750 & 12.8230263997 & 0.0364954745 \\
\hline N10 & 2.9123280287 & 13.7113486980 & 2.0639202264 \\
\hline $\mathrm{C} 12$ & 4.7871839944 & 16.3461289998 & 3.5479894747 \\
\hline
\end{tabular}




\begin{tabular}{|c|c|c|c|}
\hline H13 & 4.2451235324 & 16.1300906205 & 4.4606576851 \\
\hline $\mathrm{C} 14$ & 5.1162690058 & 17.6403631930 & 3.1312801434 \\
\hline H15 & 4.8455531335 & 18.4732263395 & 3.7673084628 \\
\hline $\mathrm{C} 16$ & 5.7578787714 & 17.8509014228 & 1.9166116219 \\
\hline H17 & 6.0006030889 & 18.8518220720 & 1.5783133156 \\
\hline $\mathrm{C} 18$ & 6.0799227898 & 16.7318459438 & 1.1454521747 \\
\hline C19 & 6.7241980284 & 16.4865164946 & -0.1126615601 \\
\hline $\mathrm{H} 20$ & 7.1203460058 & 17.2221002983 & -0.7942365374 \\
\hline $\mathrm{C} 21$ & 6.7389040953 & 15.1335829583 & -0.2941192417 \\
\hline $\mathrm{H} 22$ & 7.1401163431 & 14.5395472826 & -1.0992029115 \\
\hline $\mathrm{C} 23$ & 5.7228317961 & 15.4630417985 & 1.6699127321 \\
\hline $\mathrm{C} 24$ & 6.1557514874 & 13.0804669348 & 1.0073715396 \\
\hline $\mathrm{C} 25$ & 7.2718586486 & 12.5145892143 & 1.6237945737 \\
\hline H26 & 8.0417897164 & 13.1696473585 & 2.0068492609 \\
\hline $\mathrm{C} 27$ & 7.3604486846 & 11.1368889153 & 1.7684779478 \\
\hline $\mathrm{H} 28$ & 8.2195612942 & 10.7054167414 & 2.2668378011 \\
\hline $\mathrm{C} 29$ & 6.3373452848 & 10.3178744365 & 1.2951801016 \\
\hline $\mathrm{H} 30$ & 6.4001919220 & 9.2431791552 & 1.4178620393 \\
\hline C31 & 5.2066866180 & 10.8763985249 & 0.7139144613 \\
\hline H32 & 4.3711132234 & 10.2604460626 & 0.4073388013 \\
\hline C33 & 5.1052797436 & 12.2579954738 & 0.5756999741 \\
\hline C34 & 3.4832998914 & 12.6840028586 & -1.2809897961 \\
\hline H35 & 4.1391842732 & 12.2268679876 & -2.0043544667 \\
\hline C36 & 2.2301603045 & 13.2038210038 & -1.4224524078 \\
\hline H37 & 1.6595379598 & 13.2446263270 & -2.3363298445 \\
\hline C38 & 1.8293470039 & 13.6850763344 & -0.1319114997 \\
\hline C39 & 0.6923173233 & 14.2957548154 & 0.4014131385 \\
\hline $\mathrm{H} 40$ & -0.1643158070 & 14.5221204414 & -0.2232560338 \\
\hline $\mathrm{C} 41$ & 0.6942772409 & 14.5961202920 & 1.7564030613 \\
\hline $\mathrm{H} 42$ & -0.1634448573 & 15.0605014161 & 2.2255418171 \\
\hline $\mathrm{C} 43$ & 1.8058811232 & 14.2968457847 & 2.5501527487 \\
\hline H44 & 1.8507074095 & 14.5204463012 & 3.6081669307 \\
\hline $\mathrm{C} 45$ & 2.9033237407 & 13.4233868152 & 0.7574054434 \\
\hline H46 & 4.6778237943 & 11.3381428417 & 4.2340004364 \\
\hline $\mathrm{H} 47$ & 5.9841768463 & 13.6136298808 & 5.0739395027 \\
\hline $\mathrm{O} 45$ & 1.4658700847 & 11.4398995440 & 4.0491731961 \\
\hline $\mathrm{H} 48$ & 1.2722728516 & 10.6828784681 & 4.6138985933 \\
\hline $\mathrm{H} 49$ & 2.7947643566 & 11.3761290975 & 3.7670645118 \\
\hline $\mathrm{O} 48$ & 2.0352387520 & 13.5268489242 & 5.6875909387 \\
\hline H50 & 1.6639387030 & 12.4808551656 & 4.9016907788 \\
\hline H51 & 2.7019009177 & 13.1372075290 & 6.2608869357 \\
\hline \multicolumn{4}{|c|}{$5 \mathrm{FP}-\mathrm{Co}(\mathrm{OH})_{2}(\mathrm{O})-2\left(\mathrm{H}_{2} \mathrm{O}\right)-$ Path 4 TS } \\
\hline Co1 & 4.1366602519 & 13.2673125598 & 3.6669027345 \\
\hline $\mathrm{O} 2$ & 3.8665344104 & 11.4752814894 & 3.1088096492 \\
\hline $\mathrm{O} 3$ & 5.4214374613 & 12.7399059299 & 4.9604527257 \\
\hline $\mathrm{O} 4$ & 3.3170228071 & 14.3146454702 & 4.8043509515 \\
\hline N7 & 5.3400255361 & 15.1939513689 & 2.8202207891 \\
\hline N8 & 6.1706827448 & 14.4685069746 & 0.6755111574 \\
\hline N9 & 3.9975781580 & 12.7843717149 & 0.0806065999 \\
\hline N10 & 2.8858888195 & 13.7966537934 & 2.0410303824 \\
\hline $\mathrm{C} 12$ & 5.0686629874 & 16.2964604844 & 3.5397740897 \\
\hline H13 & 4.6736197500 & 16.1010999911 & 4.5266261251 \\
\hline $\mathrm{C} 14$ & 5.2258862563 & 17.6034624677 & 3.0579201866 \\
\hline H15 & 4.9754045285 & 18.4332857036 & 3.7095382742 \\
\hline $\mathrm{C} 16$ & 5.6784245738 & 17.8256244971 & 1.7599897161 \\
\hline
\end{tabular}




$\begin{array}{lccc}\text { H17 } & 5.7901522185 & 18.8322021371 & 1.3667615925 \\ \text { C18 } & 5.9853361612 & 16.7037572687 & 0.9844030820 \\ \text { C19 } & 6.4869407628 & 16.4669256986 & -0.3402011707 \\ \text { H20 } & 6.7335177551 & 17.2088622085 & -1.0869086940 \\ \text { C21 } & 6.5906742238 & 15.1102899790 & -0.4887431237 \\ \text { H22 } & 6.9364892550 & 14.5210221035 & -1.3262457746 \\ \text { C23 } & 5.8020132226 & 15.4271241804 & 1.5905210577 \\ \text { C24 } & 6.2757753158 & 13.0657958182 & 0.9165562384 \\ \text { C25 } & 7.4572888487 & 12.5331051365 & 1.4335647625 \\ \text { H26 } & 8.2639848049 & 13.2054023625 & 1.7062281308 \\ \text { C27 } & 7.5932452504 & 11.1532187146 & 1.5584616350 \\ \text { H28 } & 8.5149205227 & 10.7356121901 & 1.9495198206 \\ \text { C29 } & 6.5535770632 & 10.3097427813 & 1.1587525434 \\ \text { H30 } & 6.6676045973 & 9.2334224897 & 1.2340604009 \\ \text { C31 } & 5.3606259965 & 10.8441304773 & 0.6829921303 \\ \text { H32 } & 4.5320495929 & 10.2038493737 & 0.4058382771 \\ \text { C33 } & 5.2103234801 & 12.2257682881 & 0.5868076968 \\ \text { C34 } & 3.6645298550 & 12.7033121741 & -1.2727902819 \\ \text { H35 } & 4.3376353800 & 12.1952909613 & -1.9488639404 \\ \text { C36 } & 2.4875111779 & 13.3485111567 & -1.5146820679 \\ \text { H37 } & 1.9994714165 & 13.4555377128 & -2.4737183871 \\ \text { C38 } & 2.0365689496 & 13.8636703984 & -0.2554583827 \\ \text { C39 } & 0.9198843693 & 14.5994375804 & 0.1461649174 \\ \text { H40 } & 0.1668864330 & 14.9070977144 & -0.5742371807 \\ \text { C41 } & 0.8097129823 & 14.9191169938 & 1.4937964545 \\ \text { H42 } & -0.0354621416 & 15.4844090518 & 1.8700378322 \\ \text { C43 } & 1.7965705725 & 14.5004815528 & 2.3911932363 \\ \text { H44 } & 1.7534587210 & 14.7123817275 & 3.4539201003 \\ \text { C45 } & 2.9984936241 & 13.4895757982 & 0.7345231933 \\ \text { H46 } & 4.5073552523 & 10.9712499687 & 3.6311852725 \\ \text { H47 } & 4.9968828873 & 13.0973033230 & 5.7565010778 \\ \text { O45 } & 1.4314582175 & 11.6861645321 & 4.7736072287 \\ \text { H48 } & 2.1644815459 & 11.4273012433 & 4.1867067747 \\ \text { O48 } & 2.8887214178 & 13.2946906584 & 6.4101976897 \\ \text { H50 } & 1.9149171586 & 12.2972664311 & 5.3970188746 \\ \text { H49 } & 2.2770524747 & 14.0188883501 & 6.5960541856 \\ & & & \end{array}$




\section{Supplementary Figures}

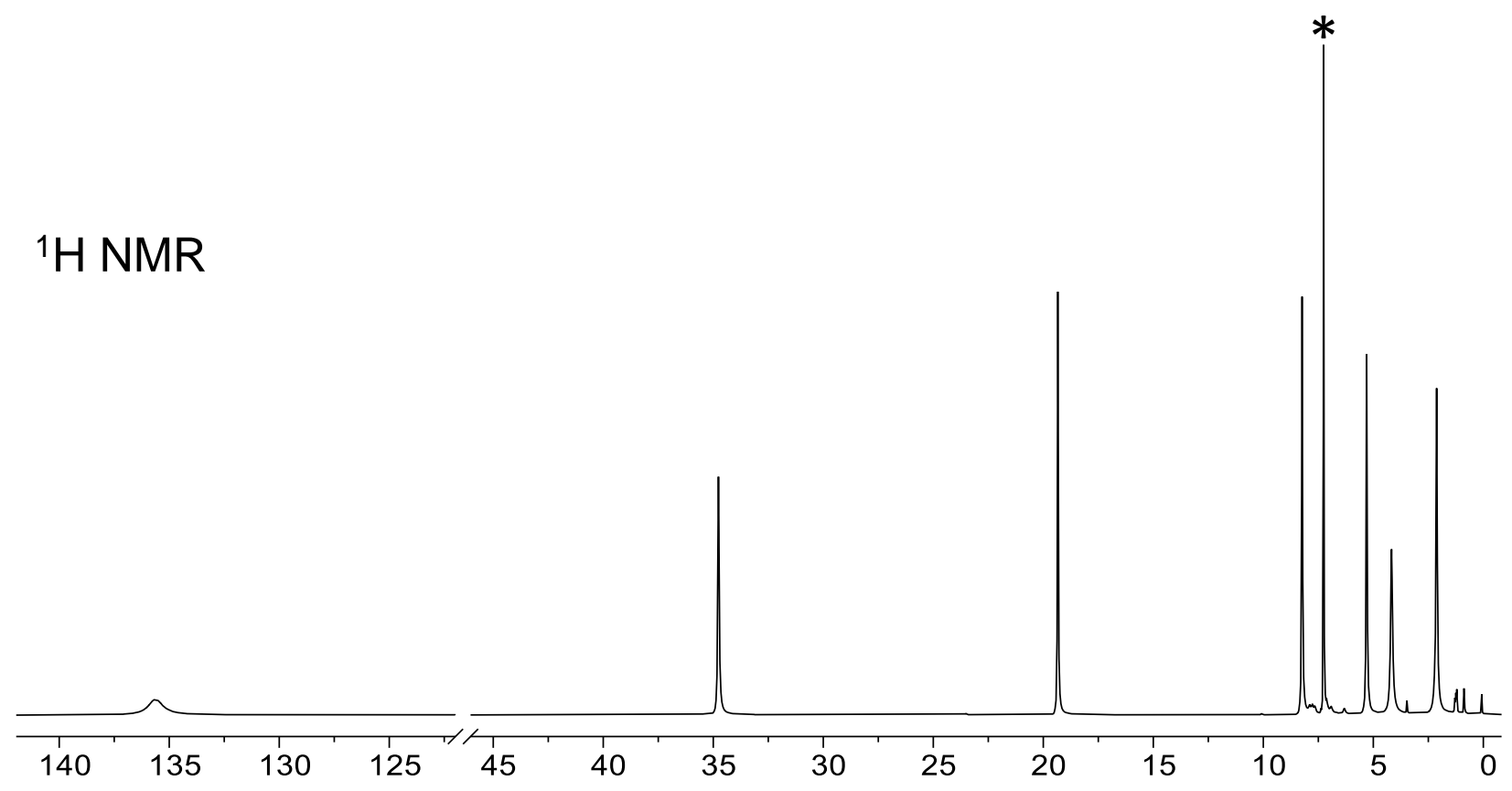

Figure S1. ${ }^{1} \mathrm{H}$ NMR spectrum of $(5-\mathrm{FP}) \mathrm{Co}\left(\mathrm{NO}_{3}\right)_{2}(2)$ in $\mathrm{CDCl}_{3}$. Indicates $\mathrm{CDCl}_{3}$ in spectra.

(A) ${ }^{13} \mathrm{C}$ NMR

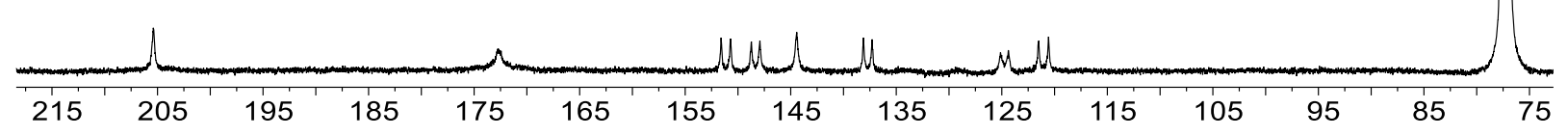

(B) ${ }^{13} \mathrm{C}$ NMR

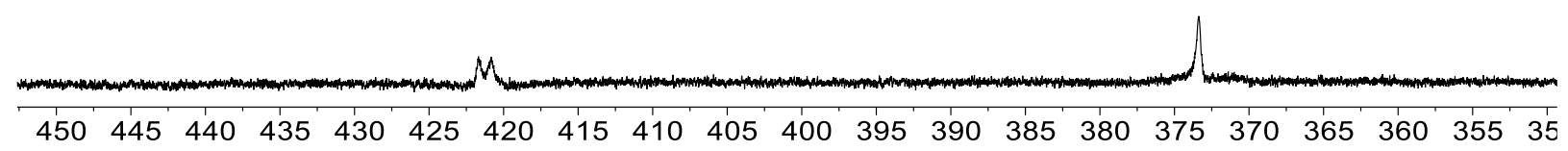

Figure S2. (A) ${ }^{13} \mathrm{C}$ NMR spectrum of $(5-\mathrm{FP}) \mathrm{Co}\left(\mathrm{NO}_{3}\right)_{2}$ (2) in the range of 75 to $220 \mathrm{ppm}$. (B) ${ }^{13} \mathrm{C}$ NMR spectrum of (5-FP) Co(NO $)_{2}(2)$ in the range of 350 to $455 \mathrm{ppm}$ in $\mathrm{CDCl}_{3}$. $*$ Indicates $\mathrm{CDCl}_{3}$ in spectra. 


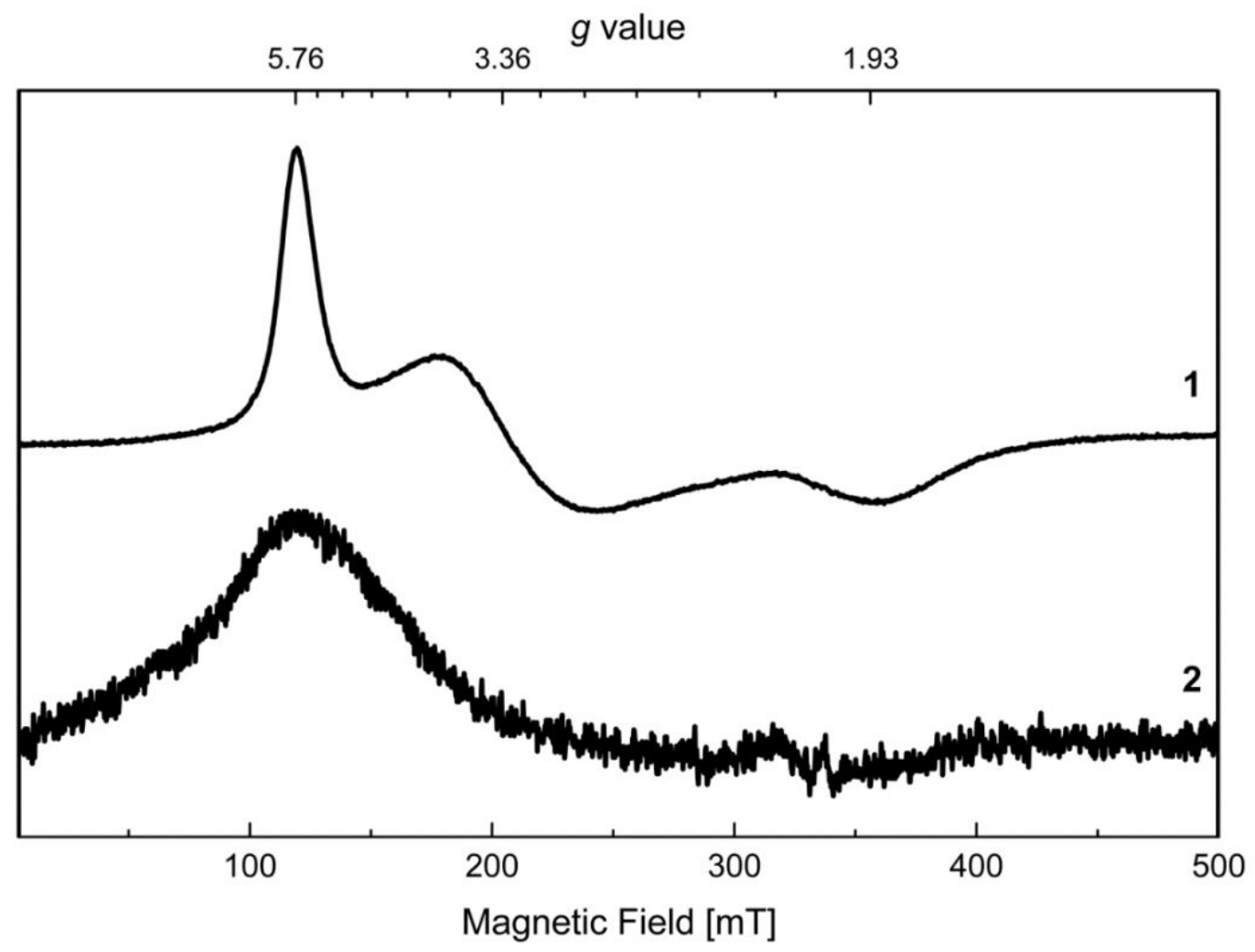

Figure S3. X-band cw-EPR spectra obtained at $10 \mathrm{~K}$ for $(6-\mathrm{FP}) \mathrm{Co}\left(\mathrm{NO}_{3}\right)_{2}(\mathbf{1})$ and $(5-\mathrm{FP}) \mathrm{Co}\left(\mathrm{NO}_{3}\right)_{2}$ (2) dissolved in a 2:1 solvent mixture of toluene and $\mathrm{CHCl}_{3}$. Complex 1 showed an EPR spectrum with resonances at effective g-values, $g_{\text {eff }}=5.76,3.36$ and 1.93 while complex 2 showed rather broad signal with peaks at $\mathrm{g}_{\mathrm{eff}}=5.76$ and 1.93 .

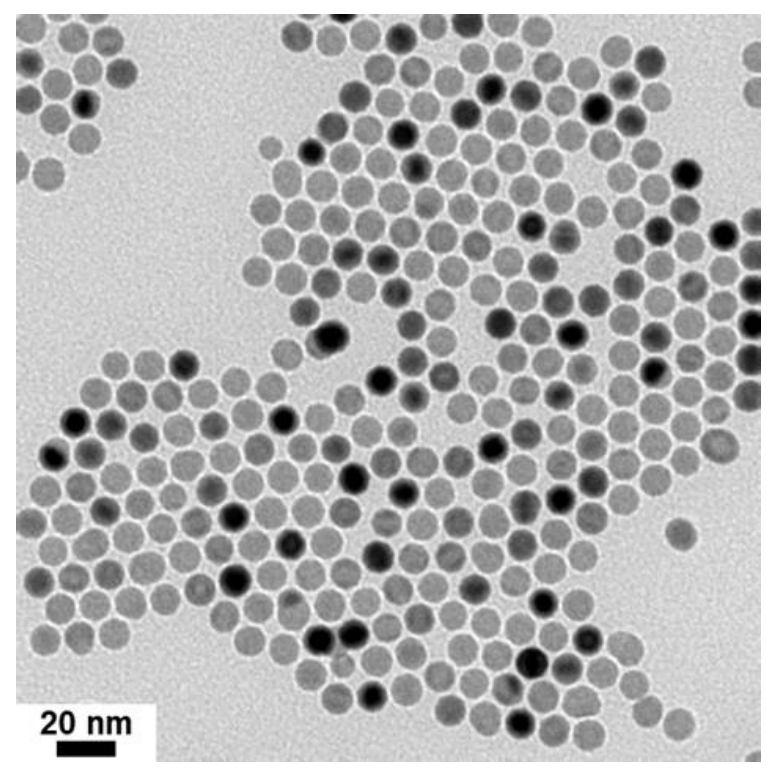

Figure S4. TEM image of monodisperse $\mathrm{Fe}_{3} \mathrm{O}_{4}$ nanoparticles. 


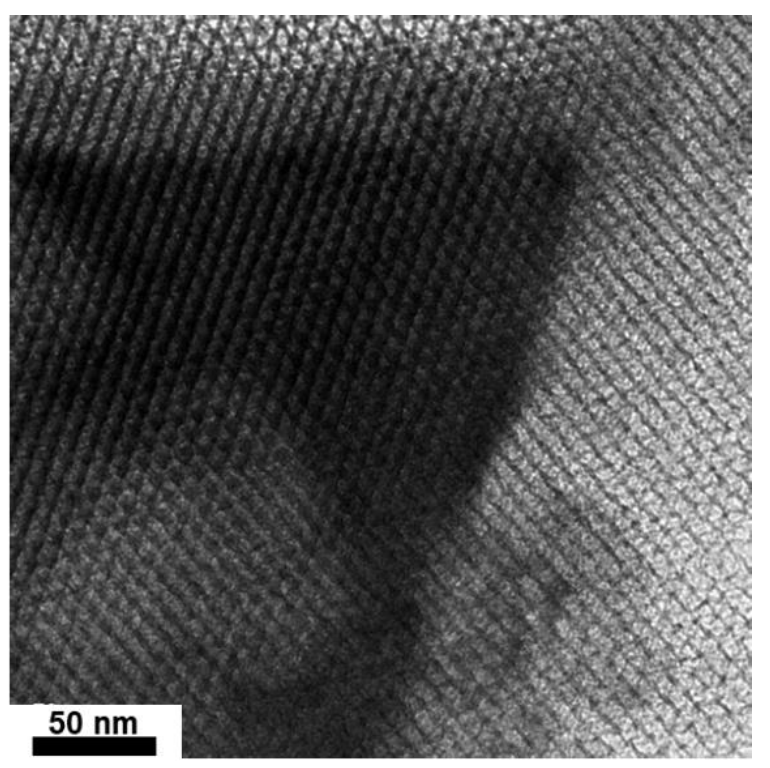

Figure S5. TEM image of OMC after $500{ }^{\circ} \mathrm{C}$ treatment.

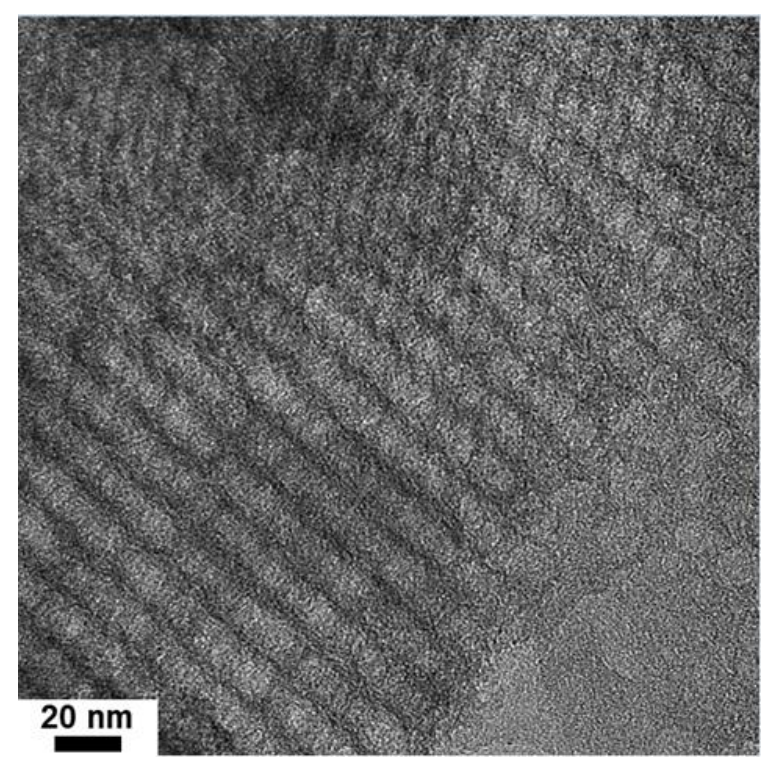

Figure S6. TEM image of 5-FP-Co-OMC-2 catalyst. 

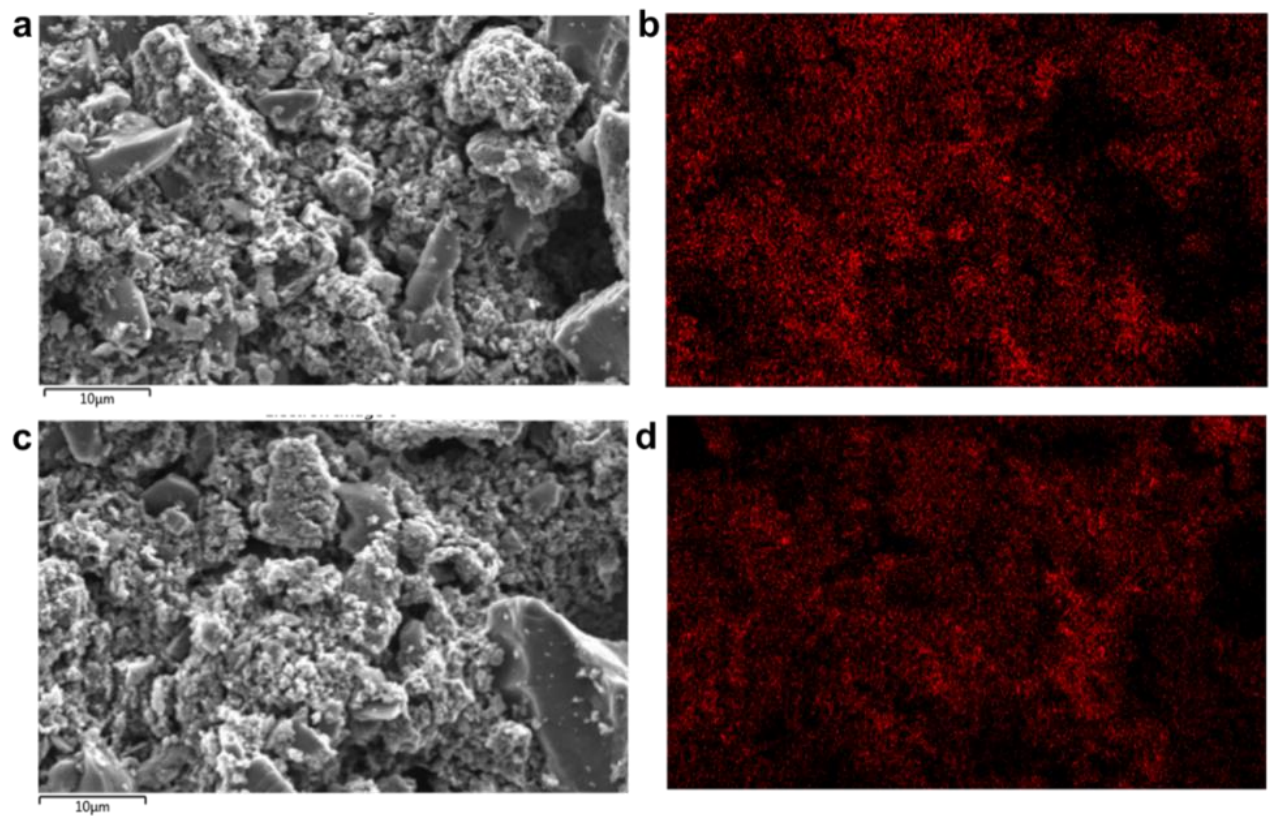

Figure S7. Elemental mapping of 6FP-Co-OMC-1 before and after OER test. (a) and (c), SEM image of 6FP-Co-OMC-1 before/after OER test. (b) and (d), Elemental mapping of Co for 6FPCo-OMC-1 before/after OER test.
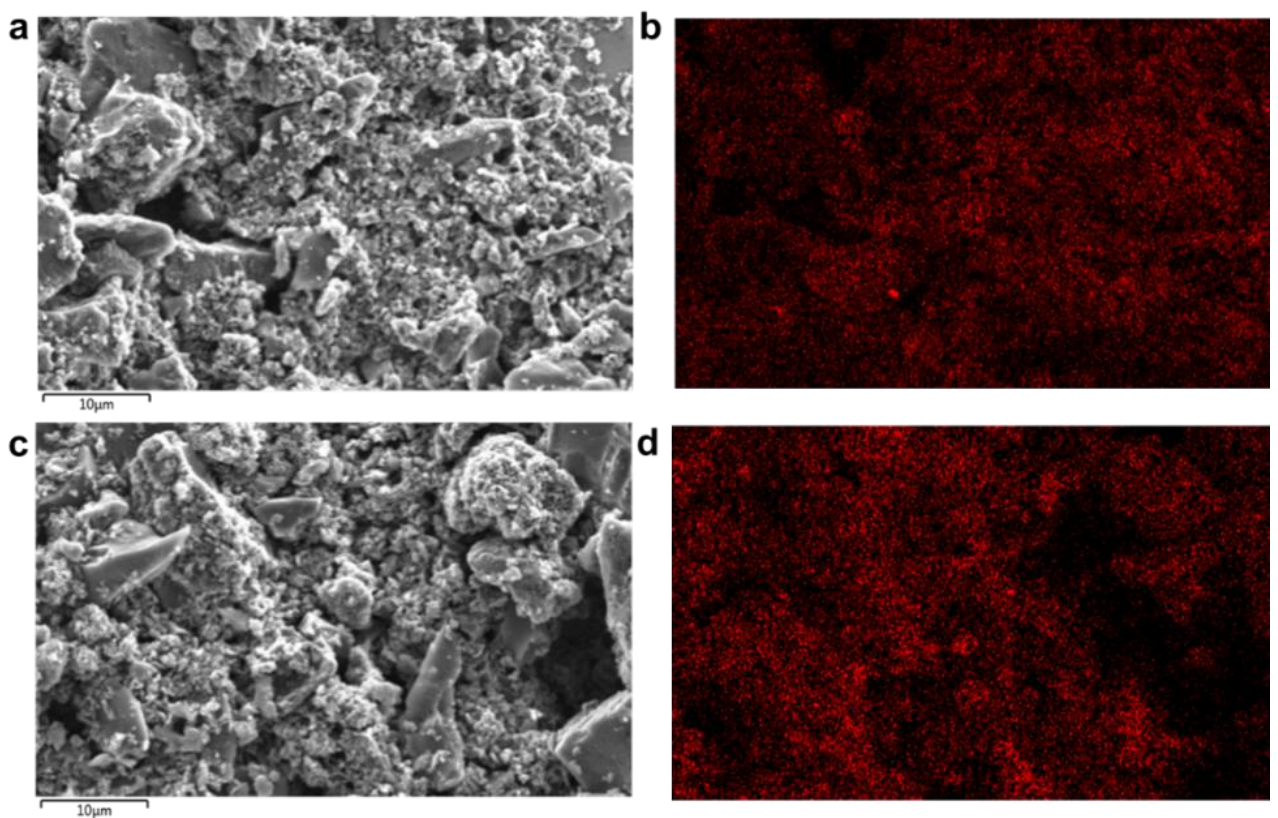

Figure S8. Elemental mapping of 5FP-Co-OMC-2 before and after OER test. (a) and (c), SEM image of 5FP-Co-OMC-2 before/after OER test. (b) and (d), Elemental mapping of Co for 5FPCo-OMC-2 before/after OER test. 


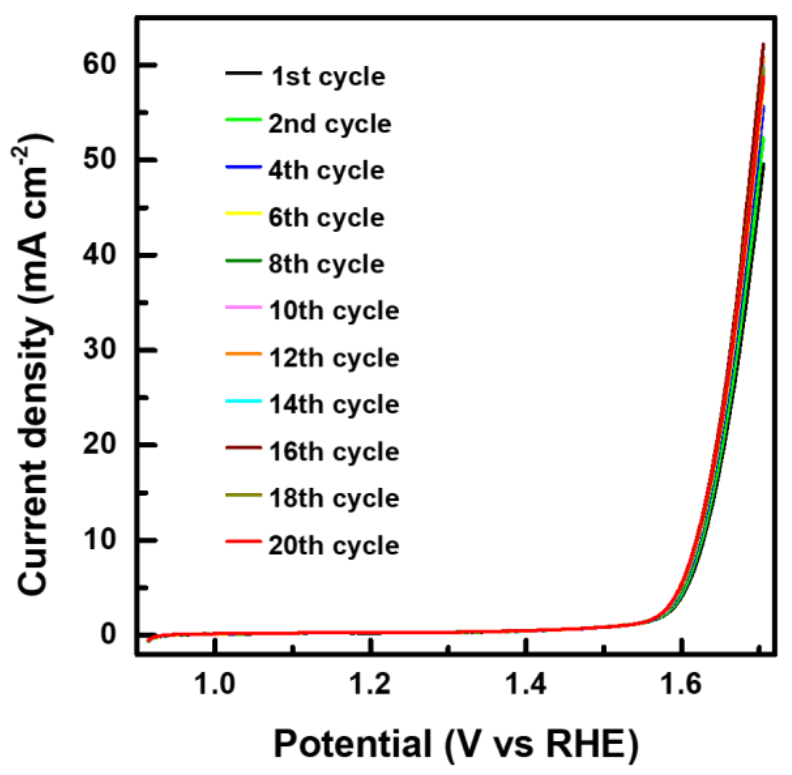

Figure S9. Multiple LSV curves (20 continuous scans) of 6-FP-Co-OMC-1 in $1.0 \mathrm{M} \mathrm{KOH}$ electrolyte at $10 \mathrm{mV} \mathrm{s}^{-1}$.

a

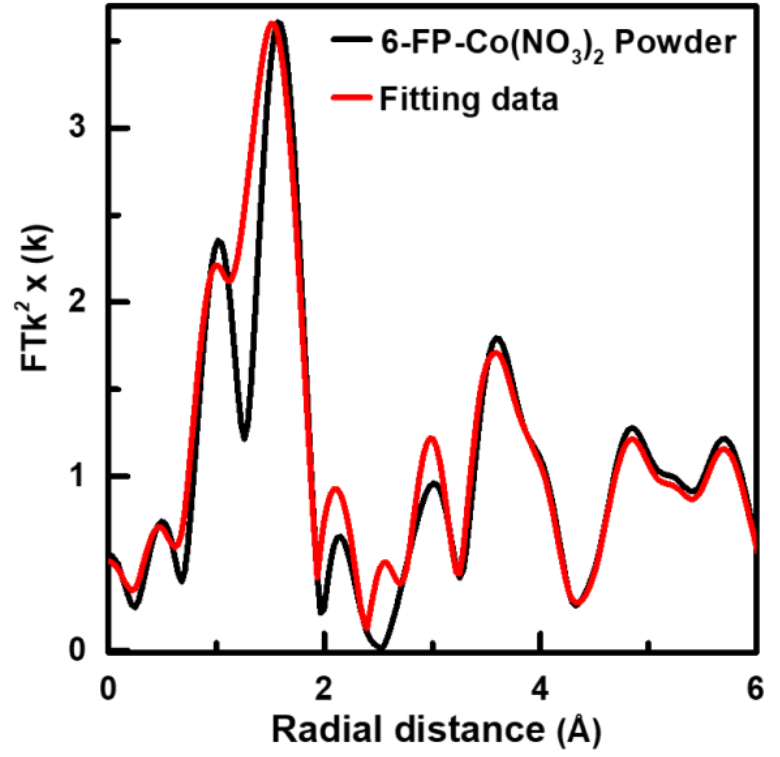

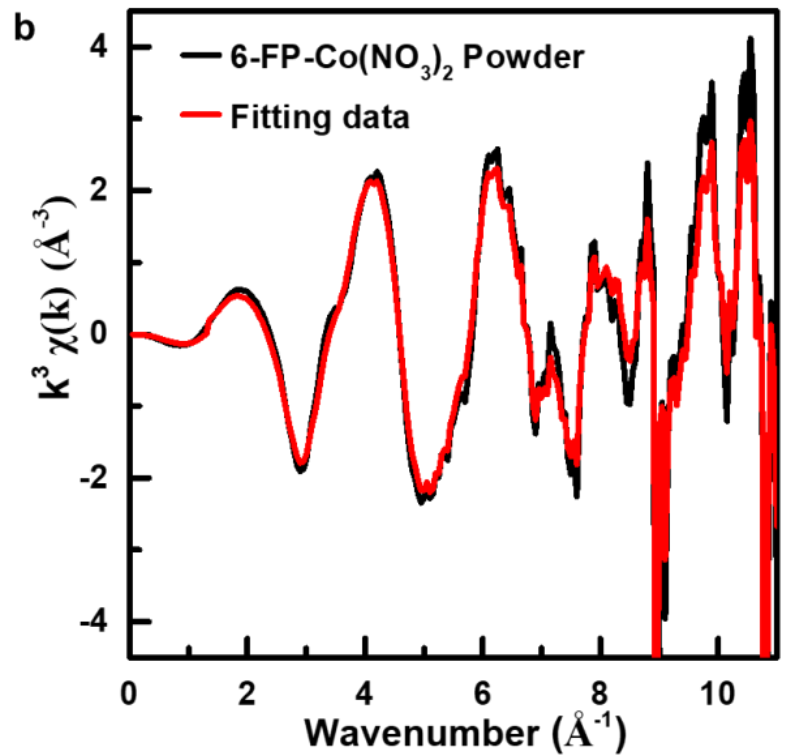

Figure S10. Co K-edge EXAFS spectra fittings for (6-FP)Co( $\left.\mathrm{NO}_{3}\right)_{2}(\mathbf{1})$. a, Fitting in R-space. b, Fitting in k-space. 

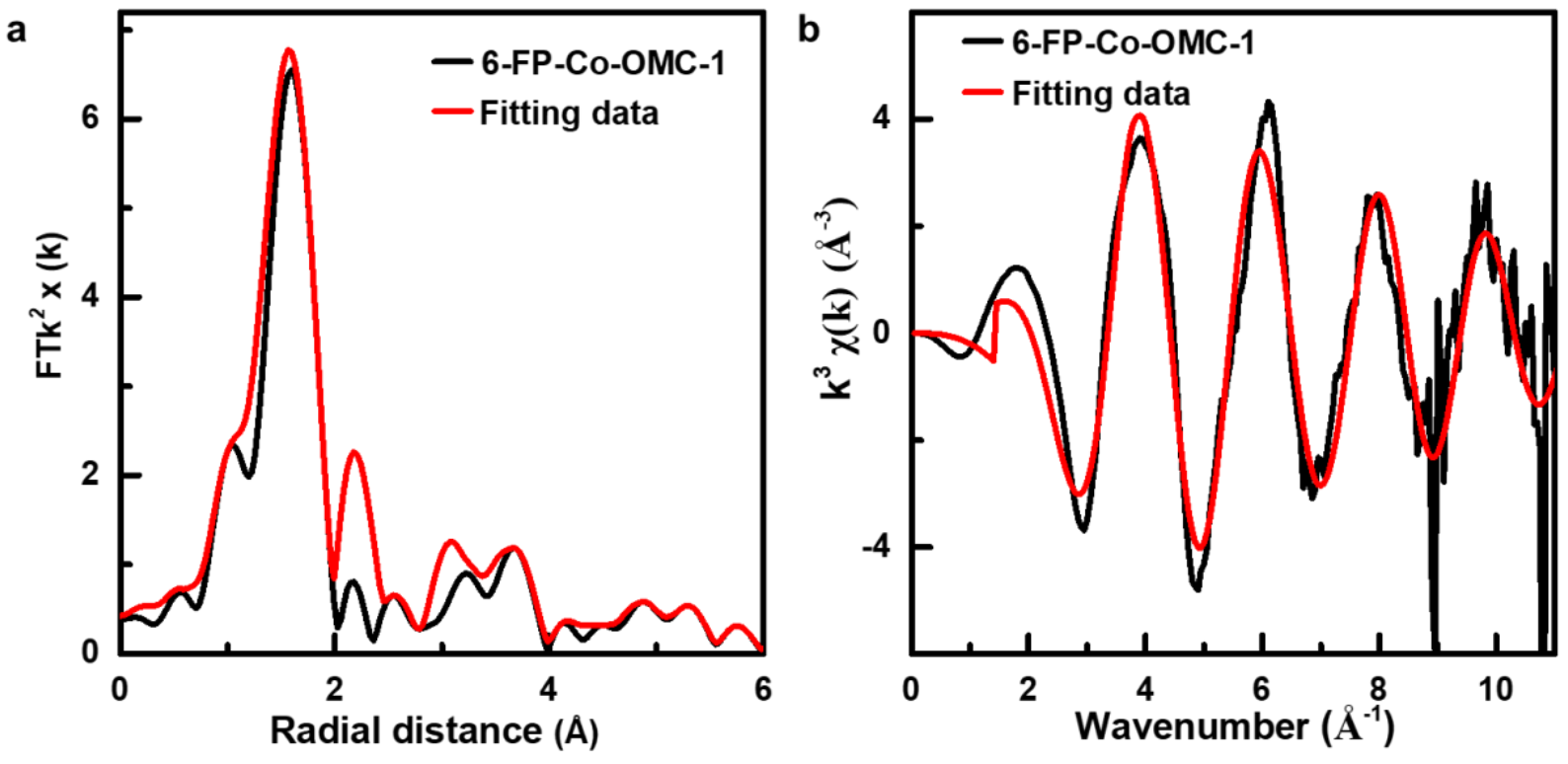

Figure S11. Co K-edge EXAFS spectra fittings for catalyst 6-FP-Co-OMC-1 before Echem. a, Fitting in R-space. b, Fitting in k-space.
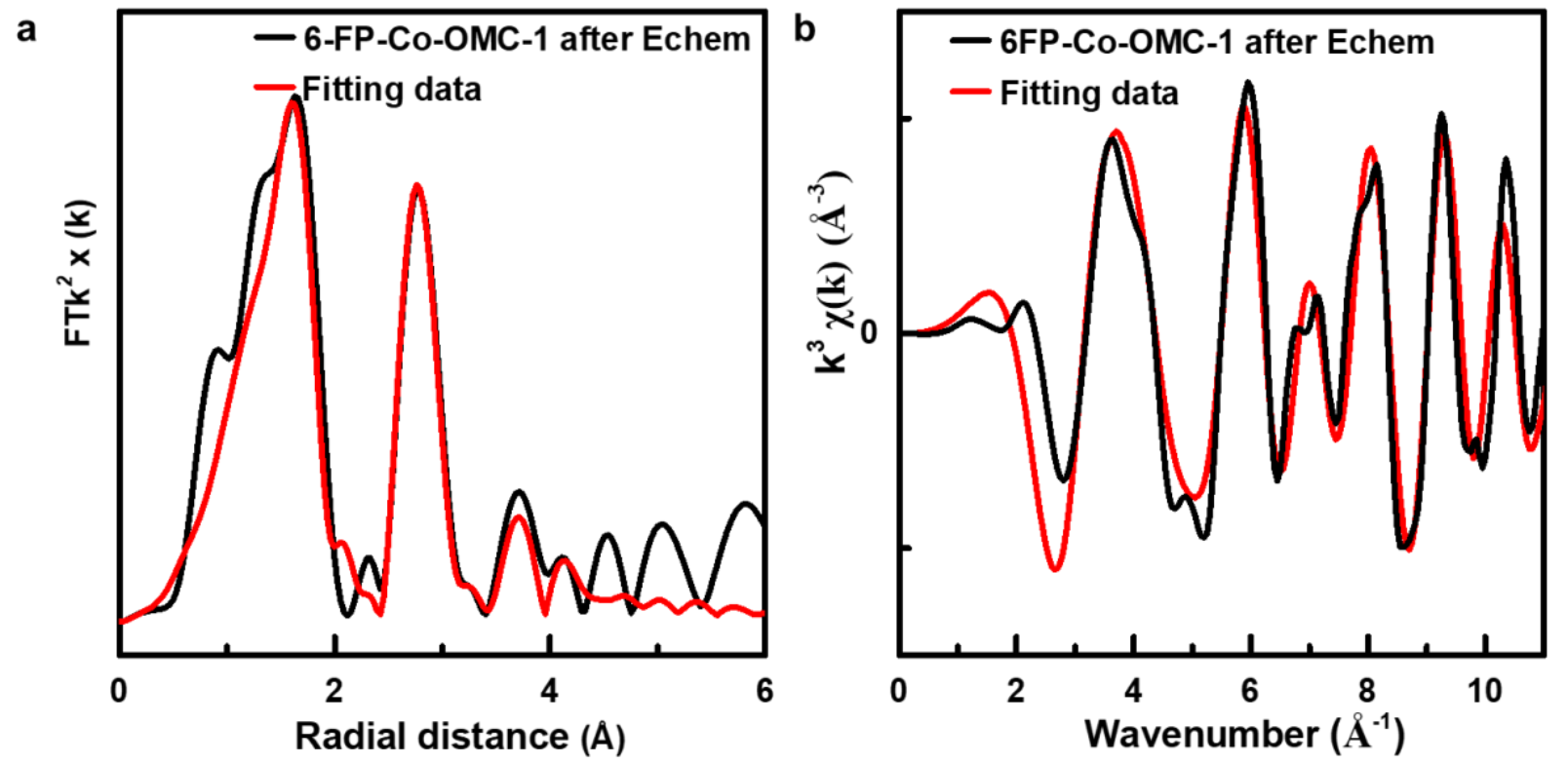

Figure S12. Co K-edge EXAFS spectra fittings for catalyst 6-FP-Co-OMC-1 after Echem. a, Fitting in R-space. b, Fitting in k-space. 


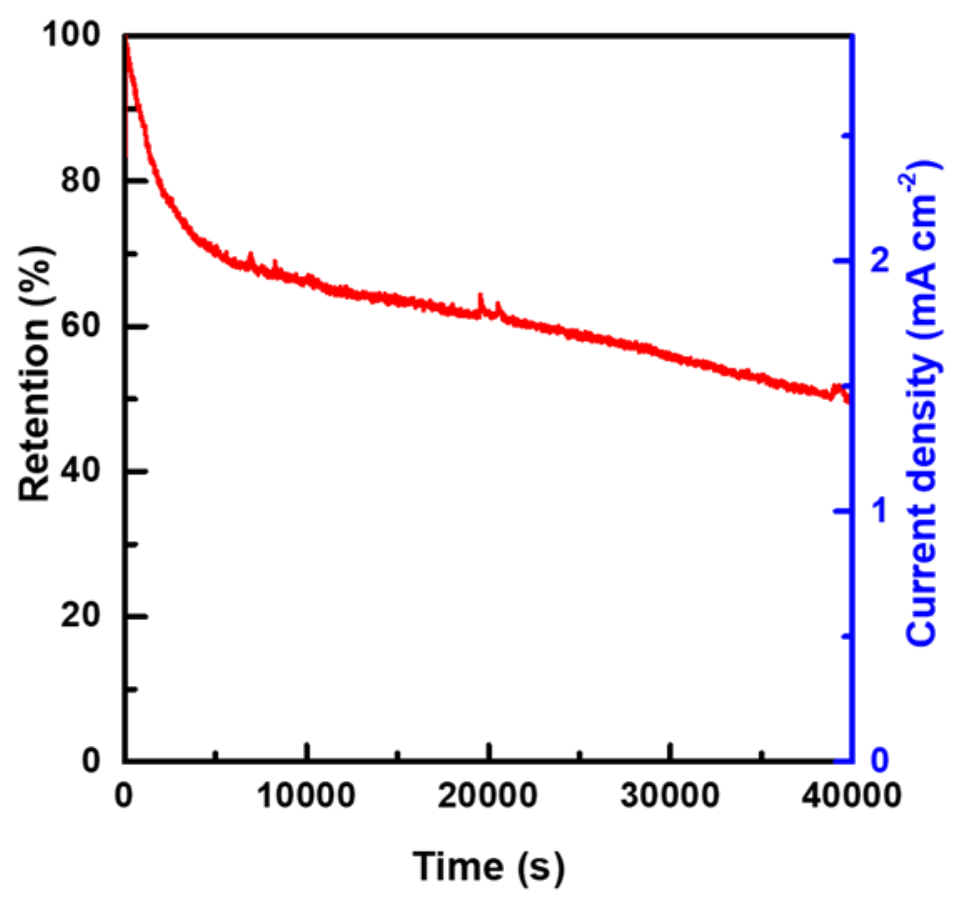

Figure S13. Stability test of 5-FP-Co-OMC-2 with chronoamperometry test.

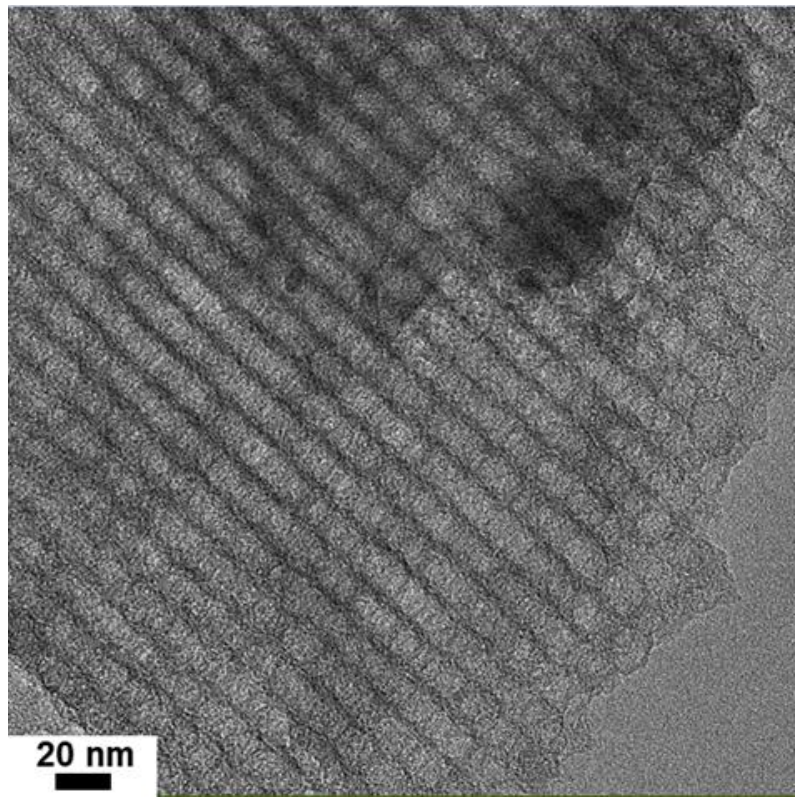

Figure S14. TEM image of 5-FP-Co-OMC-2 after OER test. 


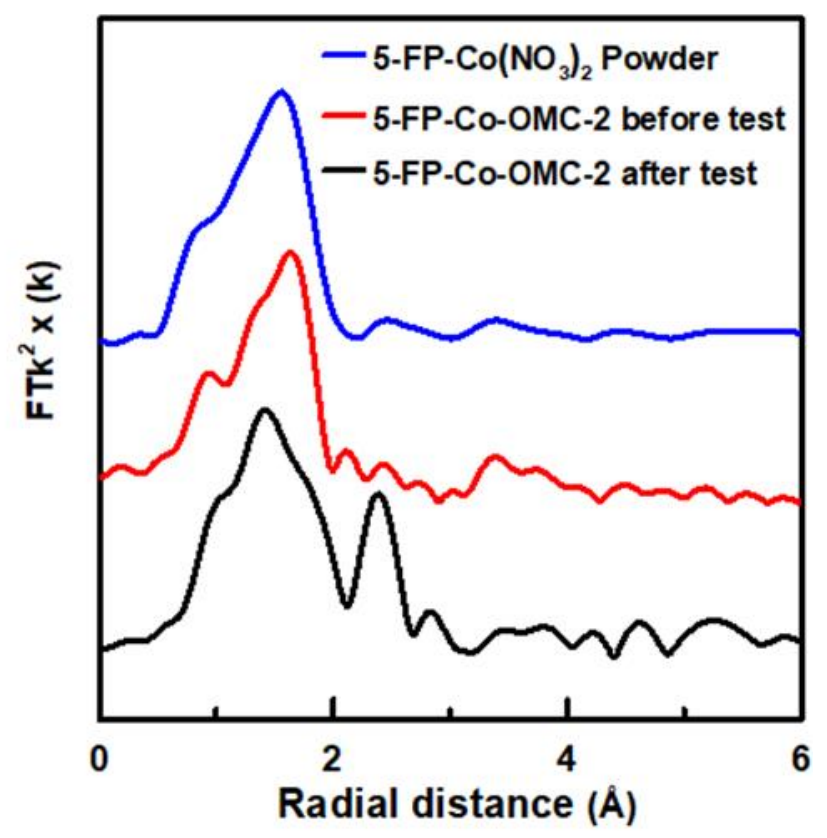

Figure S15. Ex-situ EXAFS analysis of (5-FP)Co( $\left.\mathrm{NO}_{3}\right)_{2}(2)$ and 5-FP-Co-OMC-2 for before and after electrochemical tests.
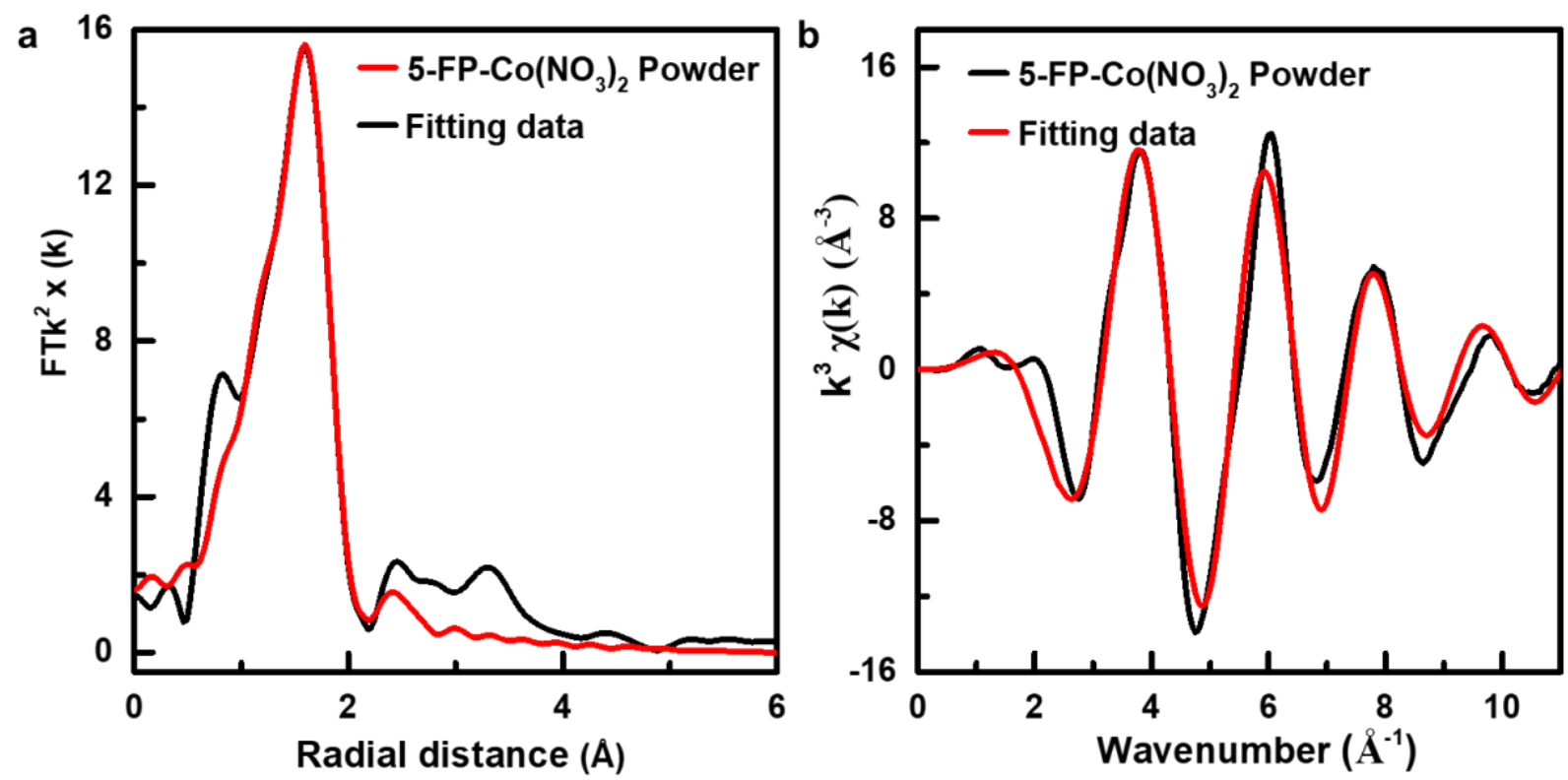

Figure S16. Co K-edge EXAFS spectra fittings for (5-FP)Co( $\left(\mathrm{NO}_{3}\right)_{2}$ (2). a, Fitting in R-space. b, Fitting in k-space. 

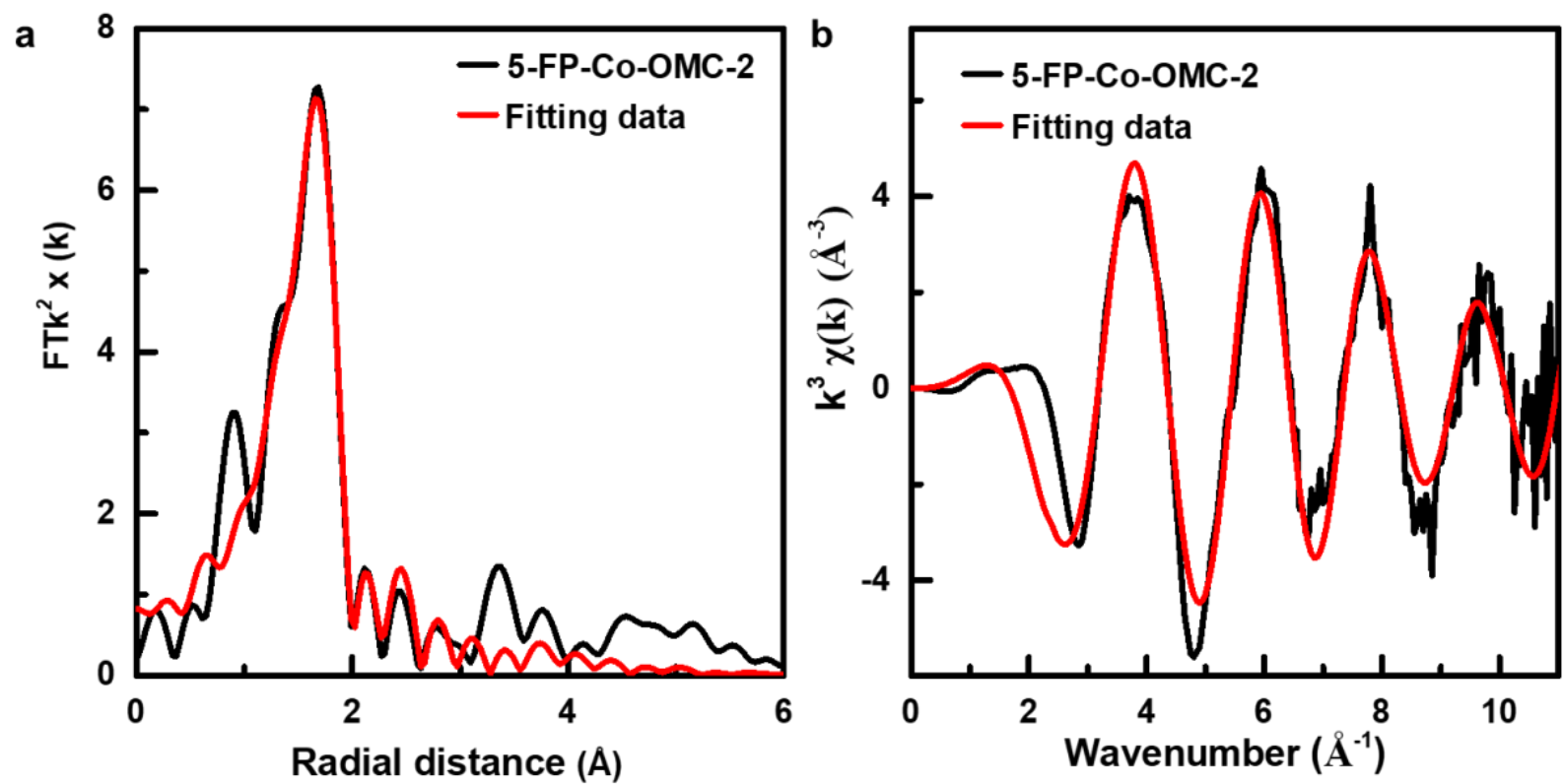

Figure S17. Co K-edge EXAFS spectra fittings for catalyst 5-FP-Co-OMC-2 before test. a, Fitting in R-space. b, Fitting in k-space.
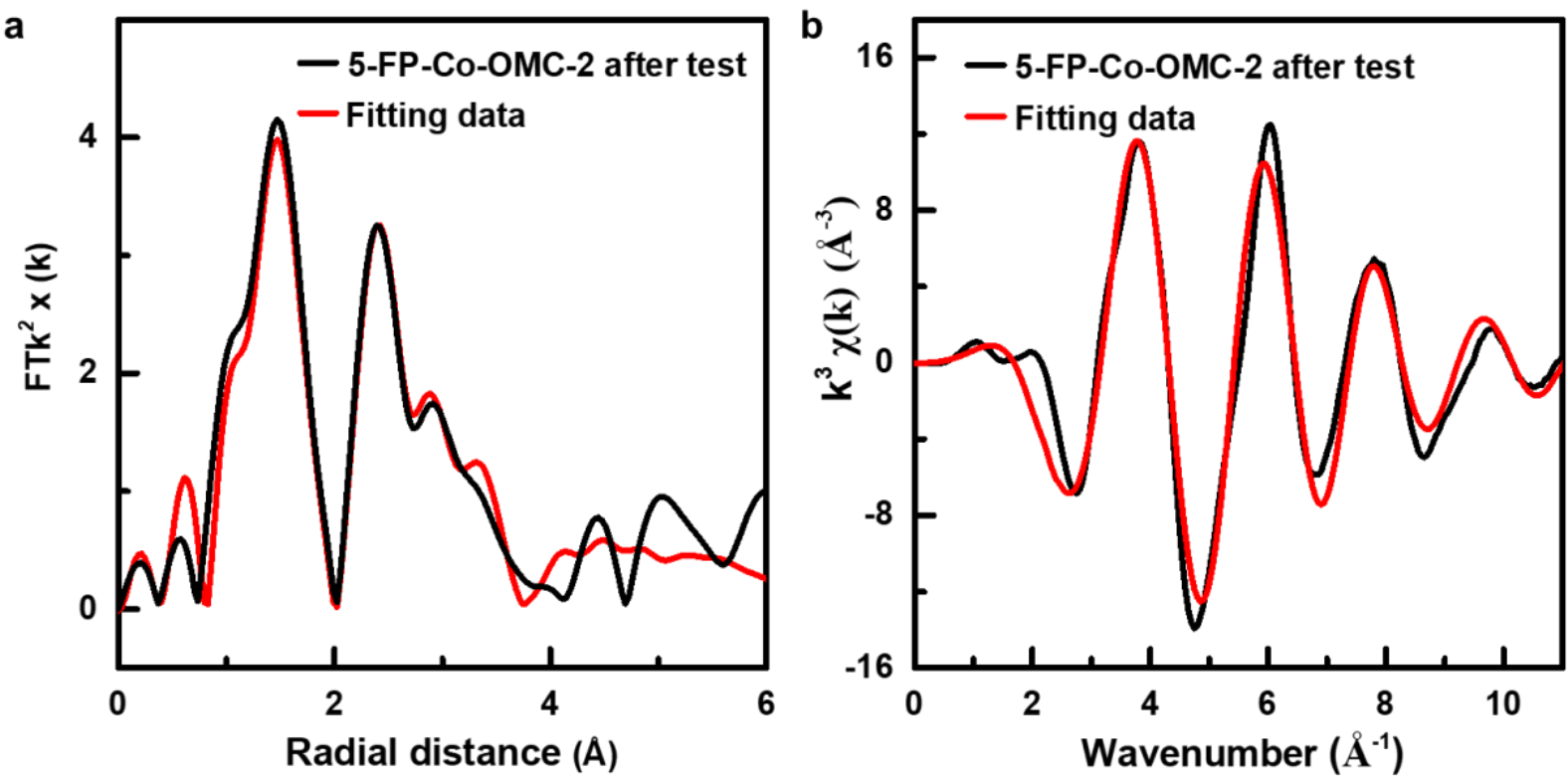

Figure S18. Co K-edge EXAFS spectra fittings for catalyst 5-FP-Co-OMC-2 after test. a, Fitting in R-space. b, Fitting in k-space. 
Table S1. Structural parameters for 6-FP-Co( $\left(\mathrm{NO}_{3}\right)_{2}$, 5-FP-Co(NO 3$)_{2}$, 6-FP-Co-OMC-1, 5-FP$\mathrm{Co}\left(\mathrm{NO}_{3}\right)_{2}$, 5-FP-Co-OMC-2 from Co K-edge for EXAFS spectra.

\begin{tabular}{|c|c|c|c|c|c|c|}
\hline Sample & Path & $\mathrm{CN}$ & $\mathbf{R}(\mathbf{A})$ & $\Delta R(\AA)$ & $\sigma^{2}\left(10^{-3} \AA\right)$ & R-factor \\
\hline \multirow{2}{*}{$6-\mathrm{FP}-\mathrm{Co}\left(\mathrm{NO}_{3}\right)_{2}$ Powder } & Co-O & 5.4 & 1.88 & 0.058 & 0.0042 & 0.006 \\
\hline & Co-N/O & 5.6 & 2.04 & -0.15 & 0.00094 & 0.02 \\
\hline \multirow{2}{*}{$\begin{array}{c}\text { 6-FP-Co-OMC-1 } \\
\text { Before test }\end{array}$} & $\mathrm{Co}-\mathrm{O}$ & 5.6 & 2.04 & 0.220 & 0.0058 & 0.009 \\
\hline & Co-N/O & 5.7 & 2.72 & 0.18 & 0.00048 & 0.01 \\
\hline \multirow{3}{*}{$\begin{array}{c}\text { 6-FP-Co-OMC-1 } \\
\text { After test }\end{array}$} & $\mathrm{Co}-\mathrm{O}$ & 5.7 & 1.88 & 0.055 & 0.0052 & 0.004 \\
\hline & Co-N/O & 5.8 & 2.15 & -0.04 & 0.0034 & 0.006 \\
\hline & Co-Co & 11.5 & 3.18 & 0.17 & 0.0075 & 0.01 \\
\hline \multirow{2}{*}{ 5-FP-Co(NO3)2 Powder } & Co-O & 5.5 & 1.86 & -0.16 & 0.025 & 0.006 \\
\hline & Co-N & 5.7 & 1.97 & -0.10 & 0.003 & 0.007 \\
\hline \multirow{2}{*}{$\begin{array}{c}\text { 5-FP-Co-OMC-1 } \\
\text { Before test }\end{array}$} & Co-O & 5.6 & 1.86 & 0.028 & 0.0086 & 0.006 \\
\hline & Co-N & 5.7 & 2.15 & 0.075 & 0.0035 & 0.01 \\
\hline \multirow{3}{*}{$\begin{array}{c}\text { 5-FP-Co-OMC-1 } \\
\text { After test }\end{array}$} & Co-O & 5.6 & 2.06 & 0.028 & 0.0086 & 0.01 \\
\hline & Co-N/O & 6.0 & 2.17 & 0.077 & 0.016 & 0.016 \\
\hline & Co-Co & 11.3 & 2.83 & -0.18 & 0.005 & 0.025 \\
\hline
\end{tabular}

\section{References}

1. Saint, B. SADABS, APEX3. Bruker AXS Inc. Madison, Wisconsin, USA 2012.

2. Lyford, T. S.; Collins, S.P.; Fewster, P. F.; Thomas, P. A. X-ray investigation of lateral hetero-structures of inversion domains in $\mathrm{LiNbO}_{3}, \mathrm{KTiOPO}_{4}$ and $\mathrm{KTiOAsO}_{4}$. Acta Crystallogr. A 2015, 71, 255-267.

3. Dolomanov, O. V.; Bourhis, L.J.; Gildea, R. J.; Howard, J. A. K.; Puschmann, H. OLEX2: a complete structure solution, refinement and analysis program. J. Appl. Crystallogr 2009, 42, 339-341.

4. Spek, A. PLATON SQUEEZE: a tool for the calculation of the disordered solvent contribution to the calculated structure factors. Acta Cryst. C 2015, 71, 9-18. 